\title{
Expression and localization of rhoptry neck protein 5 in merozoites and sporozoites of Plasmodium yoelii
}

\author{
Joe Kimanthi Mutungi ${ }^{\mathrm{a}, \mathrm{b}}$, Kazuhide Yahata ${ }^{\mathrm{a},{ }^{*}, \text { Miako Sakaguchi }^{\mathrm{c}} \text { and Osamu Kaneko, }}{ }^{\mathrm{a},{ }^{*}}$
}

\author{
${ }^{a}$ Department of Protozoology, Institute of Tropical Medicine (NEKKEN), Nagasaki University, Sakamoto, Nagasaki 852-8523, \\ Japan. \\ ${ }^{\mathrm{b}}$ Graduate School of Biomedical Sciences, Nagasaki University, Sakamoto, Nagasaki 852-8523, Japan. \\ ${ }^{\mathrm{c}}$ Central Laboratory, Institute of Tropical Medicine. (NEKKEN), Nagasaki University, Sakamoto, Nagasaki 852-8523, Japan.
}

* Corresponding authors: Department of Protozoology, Institute of Tropical Medicine (NEKKEN), Nagasaki University, 1-124 Sakamoto, Nagasaki 852-8523, Japan. Tel.: +81 95819 7838; fax: +81 958197805.

E-mail addresses: joemutungi@gmail.com (J.K. Mutungi), kyahata@nagasaki-u.ac.jp (K. Yahata), miako@nagasaki-u.ac.jp (M. Sakaguchi), okaneko@nagasaki-u.ac.jp (O. Kaneko).

Note: The nucleotide sequence reported in this paper is available in the GenBank ${ }^{\mathrm{TM}} / \mathrm{EMBL} / \mathrm{DDBJ}$ databases under the accession number: AB926016.

Keywords: Plasmodium yoelii; Invasion; Moving Junction; Rhoptry.

Host cell invasion by Apicomplexan parasites marks a crucial step in disease establishment and pathogenesis. The moving junction (MJ) is a conserved and essential feature among parasites of this phylum during host cell invasion, thus proteins that associate at this MJ are potential targets of drug and vaccine development. In both Toxoplasma gondii and Plasmodium falciparum, a micronemal protein, Apical Membrane Antigen 1 (AMA1), and Rhoptry Neck proteins (RONs; RON2 and RON4) form an essential complex at the MJ. A new RON member, RON5, was shown to be important to stabilize RON2 during development and to associate with the MJ complex in $T$. gondii and also to be immunoprecipitated by anti-AMA1 antibody in P. falciparum. However, the detailed molecular nature of RON5 in Plasmodium is not well understood. In this study, Plasmodium yoelii RON5 gene (pyron5) was identified as an ortholog of $P$. falciparum and Plasmodium berghei ron5. The pyron 5 exon-intron structure was validated by comparing genomic DNA sequences and experimentally determining fulllength complementary DNA sequence. PyRON5 was detected in water-insoluble fractions but no reliable transmembrane domain(s) were predicted by transmembrane prediction algorithms. PyRON5 formed a complex with $P y$ RON4, $P y$ RON2, and $P y A M A 1$ in late schizont protein extract. Taken together, we infer that these results suggest that $P y$ RON5 associates with membrane indirectly via other MJ components. Indirect immunofluorescence assay and immunoelectron microscopy localized $P y$ RON5 at the rhoptry neck of the late schizont merozoites and at the rhoptry of sporozoites. The two-stage expression of $P y$ RON5 suggests that $P y$ RON5 plays roles in invasion of not only erythrocytes, but also of mosquito salivary glands and/or mammalian hepatocytes.

\section{Graphic Abstract}

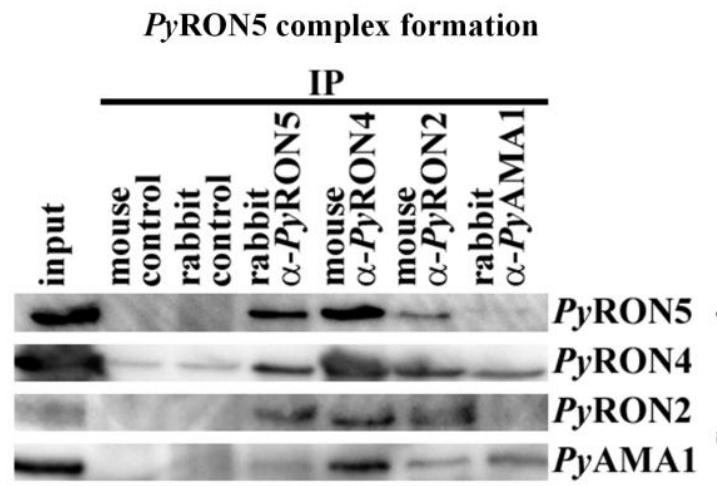

PyRON5 colocalisation with PyRON4 PyRON5 localisation by IEM
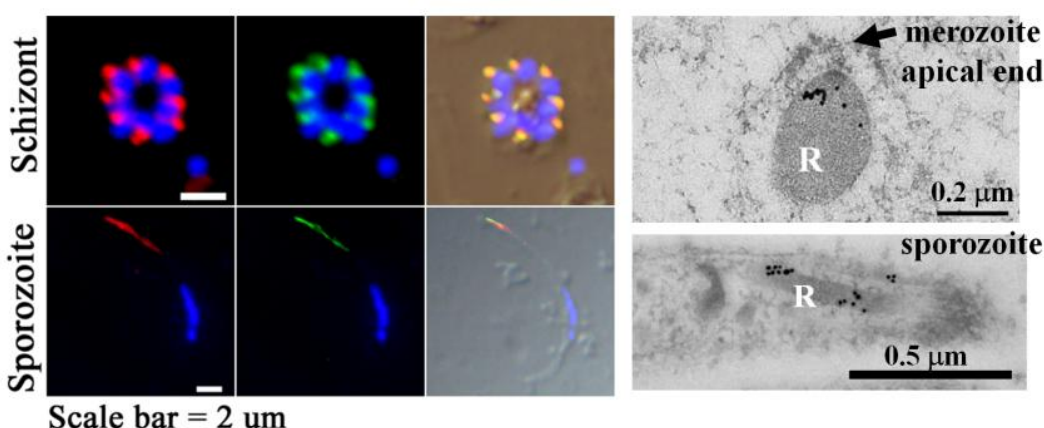

Scale bar $=2$ um

\section{Highlights}

- PyRON5 forms a complex with RON2, RON4, and AMA1 in schizont extract.

- PyRON5, a putative soluble protein, exists in the water-insoluble fractions.

- These suggest that PyRON5 associates with membrane via other complex component(s).

- PyRON5 is expressed in the rhoptry in both merozoite and sporozoite parasites.

- Targeting PyRON5 may prevent cell invasion by both merozoite and sporozoite. 


\section{Introduction}

The invasive stages of the phylum Apicomplexa, to which malaria parasites (Plasmodium spp.) and Toxoplasma gondii belong, possess a conserved apical complex containing specialized secretory organelles; micronemes, rhoptries, and dense granules, whose contents are sequentially discharged during host cell invasion [1]. The conservation of a microneme protein, Apical Membrane Antigen 1 (AMA1), and Rhoptry Neck proteins (RONs) including RON2, -4 , and -5 , in both Plasmodium and $T$. gondii suggests a conserved host-cell invasion mechanism [2 - 6]. In both parasites, AMA1 and the RONs form a complex which is involved in the establishment of a tight adhesive ring-like structure at the parasite-host cell interface, called the "moving junction (MJ)", which progresses from the anterior to the posterior of the parasite during invasion, leading to internalization of the parasite into a nascent parasitophorous vacuole (PV), within which the parasite develops to successive stages [7, 8]. The functional role of RON2 during its interaction with AMA1 at the MJ in $T$. gondii tachyzoites and $P$. falciparum merozoites and its association with both RON4 and RON5, is now understood [2, 9]. Although the topology of the RONs in the MJ in P. falciparum has not been conclusively elucidated, immunofluorescence assays along with confocal microscopy showed PfRON2, PfRON4, and PfAMA1 colocalizing at the MJ and immunoelectron microscopy (IEM) revealed PfRON4 localizing on the erythrocyte cytoplasmic side during invasion [10]. It is currently unknown what role RON5 plays in cell invasion in malaria parasites. However, the current model suggests that the RON complex is inserted into the host cell with RON2 having a surface exposed Cterminal ectodomain, part of which serves as a receptor for AMA1 on the parasite surface, while the other RONs are thought to be discharged into the host cell cytoplasm $[2,7$, 9].

Due to numerous similarities with human malaria parasites, rodent malaria parasites have been used to complement the study of the human malaria parasites infection of liver, blood, and mosquito cells [10 - 13]. Proteomic analysis demonstrated that RON2, RON4, and RON5 are present in $P$. falciparum sporozoites in both oocysts and mosquito salivary glands [14]. Myc-tagged RON2 and AMA1 were shown to localize at the rhoptries and micronemes, respectively, in the rodent malaria parasite Plasmodum berghei merozoites and sporozoites [15]. These findings, taken together with the essentiality of RON4 in $P$. berghei sporozoite invasion of hepatocytes [16], suggest a role for RON proteins in both hepatocyte and erythrocyte invasion. This implies that Plasmodium parasites could employ similar invasion strategies in two distinct life cycle stages. Radio-labelled immunoprecipitation data suggested an AMA1-RON4 interaction in Plasmodium yoelii merozoites but there is, as yet, no evidence for the presence of any potential PyRON2 and $P y$ RON5 proteins among the components of the complexes $[17,18]$.

Here, we describe the primary protein structure of $P y$ RON5 and its possible membrane association. We also show that $P y$ RON5 forms a complex with $P y$ RON2, $P y$ RON4, and PyAMA1 by immunoprecipitation of blood stage schizont protein extracts. Furthermore, we show that $P y$ RON5 is localized at the rhoptry neck in schizont stage merozoites, and in the rhoptries of sporozoites. These results suggest that PyRON5 may play a role in erythrocyte invasion and salivary gland and/or hepatocyte invasion.

\section{Materials and methods}

\subsection{Experimental animals and malaria parasites}

P. yoelii 17XL (Py17XL), obtained from Nagasaki University's BioResource bank (http://www.nbrp.jp/) was maintained in 6 to 8 week old female ICR mice (SLC Inc., Shizuoka, Japan). All experiments conducted in this study were approved by the animal care and use committee of Nagasaki University (Permit number 0912080806-4).

In order to isolate oocyst stage sporozoites, Anopheles stephensi midguts were dissected at day 9 post feed, gently crushed in sterile phosphate buffered saline (PBS) in an all plastic homogenizer, and sporozoites harvested from the supernatant after a brief low-speed centrifugation to remove mosquito debris. Similarly, salivary gland sporozoites were harvested at day 17-21 post feed by dissection and homogenisation of mosquito salivary glands in PBS.

\subsection{Extraction of parasite genomic DNA (gDNA) and $R N A$, and complementary DNA (cDNA) synthesis}

Py17XL-infected blood was collected from mice into saline sodium citrate and passed once through CF11 column (Whatman ${ }^{\circledR}$ International Ltd, UK) to remove leukocytes. The pass-through was then treated with $0.15 \%$ saponin (Wako Pure Chemical Industries, Ltd, Japan) in PBS, centrifuged, then the resultant pellet was washed in PBS. Genomic DNA was extracted using DNAzol BD reagent (Invitrogen, Carlsbad, CA). Total RNA was isolated from the pellet stored at $-80^{\circ} \mathrm{C}$ in TRIzol (Invitrogen). Complementary DNA was synthesized using SuperScript $^{\circledR}$ III Reverse Transcriptase Kit (Invitrogen) with random hexamers after DNase I treatment. To ensure no contaminating DNA remained in the cDNA, a negative control (without reverse transcriptase) was included.

\subsection{Polymerase Chain Reaction (PCR) amplification, sequencing, and analysis}

DNA fragments were PCR-amplified from gDNA and cDNA of Py17XL parasite using KOD -Plus- Neo DNA polymerase with a panel of primers (Table 1). The PCR products were treated with exonuclease I and shrimp alkaline phosphatase (ExoSAP-IT, USB Corporation, Cleveland, $\mathrm{OH}$ ), then sequenced with the same primers used for the PCR amplification and other specific primers (Table 1) using either a 3730 DNA analyzer or ABI PRISM $^{\circledR}$ 3130xl genetic analyzer (Applied Biosystems, Foster City, CA). Obtained sequences were aligned using MUSCLE webware [19]. SignalP 4.1 [20] was used to search for signal peptide sequence and the presence of transmembrane (TM) domains was evaluated with Phobius [21] and OCTOPUS [22].

\subsection{Antisera production}

Anti-PyRON5, anti-PyRON2 (PY17X 1319500, previously PY06813), and anti-PyAMA1 
(PY17X 0916500, previously PY01581) polyclonal antisera were produced by immunisation of rabbits and mice with a mixture of three synthetic peptides for each protein. A cysteine residue was added to the $\mathrm{N}$ or $\mathrm{C}$ terminus of each peptide to allow conjugation with keyhole limpet hemocyanin (KLH) for immunisation (Table S1). Rabbit antibodies that were affinity-purified using NHS-activated Sepharose ${ }^{\mathrm{TM}} 4$ Fast Flow gel (GE Healthcare Life Sciences, Sweden) with the peptide NNHRNDSKLEHSNKNTFD-C for anti-PyRON5 (amino acid positions (aa) 36-53) or CGKKGENYDRMGQADDYGKSKSR for anti-PyAMA1 (aa 505-526) were found to react most strongly by indirect immunofluorescence assay and Western blot against the protein (data not shown), and were used in the relevant experiments. Mouse anti-PyRON2 and mouse anti$P y A M A 1$ antisera were used without purification. Mouse monoclonal anti-PyRON4 (mAb 48F8) [17] was obtained from David L. Narum (National Institutes of Health, USA). Mouse monoclonal anti-Py RhopH3 (mAb\#32) has been described previously [23]

\subsection{Protein extraction from parasites, $S D S-P A G E$ and Western blotting}

Parasite-infected whole blood was obtained as in section 2.2 and the schizont-rich fraction was separated by differential centrifugation through $13.8 \mathrm{~g} / 100 \mathrm{~mL}$ Histodenz $^{\mathrm{TM}}$ (Sigma-Aldrich Co. St. Louis, MO) in PBS, $\mathrm{pH}$ 7.4. The schizonts were washed 3 times with sterile PBS supplemented with a protease inhibitor cocktail tablet, cOmplete, EDTA-free (Roche, Switzerland) and $1 \mathrm{mM}$ EDTA (PBS-PI). The pellet was then treated with $0.15 \%$ saponin in PBS as described in section 2.2 and then kept at $-80^{\circ} \mathrm{C}$ until protein extraction. Three sequential extraction procedures were used to obtain crude protein extract from the same pellet. First, the water-soluble fraction (FT) was extracted by three times repeated freeze-thaw at $-80^{\circ} \mathrm{C}$ in PBS-PI and the soluble fraction was obtained by centrifugation at $21,500 \times \mathrm{g}$ for $10 \mathrm{~min}$. The waterinsoluble pellet was washed five times with PBS-PI to avoid carry-over of any soluble extract. To verify the completion of this washing step, the freeze-thaw process was repeated once more and extract was saved for the later analysis. Proteins were further extracted in PBS-PI containing 1\% Triton X-100 (Tx; Calbiochem, San Diego, CA) for $30 \mathrm{~min}$ on ice. The Tx-insoluble pellet was washed five times with PBS-PI-Tx and pellet was once more treated with PBS-PI-Tx and extract was saved for later analysis. Protein was further extracted with PBS-PI containing $2 \%$ sodium dodecyl sulfate (SDS; Nacalai Tesque, Japan) for $30 \mathrm{~min}$ at room temperature (RT). In all extraction steps, the final parasite concentration was adjusted to $2 \times 10^{6}$ schizonts $/ \mu \mathrm{L}$. Proteins were also sequentially extracted from oocyst-derived sporozoites in similar process as for the blood stage parasites except that in this case the pellet was washed three times after each extraction step. The FT fraction from schizont extracts was also obtained by centrifugation at $132,335 \times \mathrm{g}$ for $60 \mathrm{~min}$. The protein fractions were resolved by electrophoresis on 5-20\% SDS polyacrylamide gradient mini gels (ATTO, Japan) under reducing conditions $\left(1 \times 10^{7}\right.$ schizonts or $9 \times$ $10^{3}$ sporozoites for each lane). The protein bands were transferred from gels to polyvinylidene fluoride (PVDF) membranes (Millipore, Billerica, MA). The blotted membrane was immunostained with affinity-purified rabbit anti-PyRON5, rabbit anti-PyAMA1 antibodies, or mouse anti-PyRON2 serum for $1 \mathrm{~h}$ at RT followed by secondary incubation with horseradish peroxidase (HRP)conjugated goat anti-rabbit or anti-mouse IgG (Promega, Madison, WI). Normal rabbit IgG (Millipore) or normal mouse serum were used as negative controls. In all cases, affinity-purified primary antibodies and normal IgG controls were used at a final concentration of $2 \mu \mathrm{g} / \mathrm{mL}$ while crude antisera and normal preimmune serum controls were diluted to 1:500. Bands were visualized with Immobilon $^{\text {TM }}$ Western Chemiluminescent HRP substrate (Millipore) and detected using a chemiluminescence detection system (LAS-4000EPUVmini; Fujifilm, Japan). The relative molecular sizes of the protein bands were calculated based on reference to the molecular size standards (Precision Plus Protein ${ }^{\mathrm{TM}}$ Dual Color Standards; Bio-Rad).

\subsection{Complex formation evaluation by immunoprecipitation}

Immunoprecipitation was carried out as previously described [24]. Proteins were extracted from late schizont parasite pellet once with Tx treatment. The supernatants (50 $\mu \mathrm{L}$, final $0.5 \% \mathrm{Tx}$, corresponding to $1 \times 10^{7}$ parasitized erythrocytes) were pre-incubated with $20 \mu \mathrm{L}$ of $50 \%$ protein G-conjugated beads (GammaBind Plus Sepharose; GE Healthcare) in NETT buffer $(0.15 \mathrm{M} \mathrm{NaCl}$, $1 \mathrm{mM}$ EDTA, $50 \mathrm{mM}$ Tris- $\mathrm{HCl}$, and $0.5 \% \mathrm{Tx})$ supplemented with $0.5 \%$ BSA (fraction V; Sigma) at $4{ }^{\circ} \mathrm{C}$ for $1 \mathrm{~h}$. The recovered supernatants were incubated with 2 $\mu \mathrm{L}$ of rabbit anti-PyRON5, crude mouse anti-PyRON2, rabbit anti-PyAMA1, or mouse anti-PyRON4 antibodies. Normal mouse IgG (Millipore) or rabbit preimmune serum was used as negative control. The suspensions were gently rotated at $4^{\circ} \mathrm{C}$ for $2 \mathrm{~h}$, and then $20 \mu \mathrm{L}$ of $50 \%$ protein $\mathrm{G}$ conjugated beads was added. After $1 \mathrm{~h}$ incubation at $4{ }^{\circ} \mathrm{C}$, the suspensions were centrifuged and supernatants were kept at $-80^{\circ} \mathrm{C}$ for further analysis. The beads were washed sequentially with NETT- $0.5 \%$ BSA, NETT, high-salt NETT $(0.5 \mathrm{M} \mathrm{NaCl}), \mathrm{NETT}$, and then low-salt NETT $(0.05 \mathrm{M} \mathrm{NaCl}$ and $0.17 \% \mathrm{Tx})$. Finally, proteins were extracted from the beads by boiling at $100^{\circ} \mathrm{C}$ for $3 \mathrm{~min}$ in 1x SDS-PAGE sample loading buffer (2\% SDS, 5\% 2mercaptoethanol, $10 \%$ glycerol, $0.002 \%$ bromophenol blue, $62.5 \mathrm{mM}$ Tris-HCl, $\mathrm{pH}$ 6.8). Supernatants containing immunoprecipitated proteins were collected after centrifugation and resolved on SDS-PAGE. Proteins were transferred to PVDF membranes, and then probed with the antibodies described in this section.

\subsection{Indirect Immunofluorescence assay (IFA)}

Schizont-rich whole blood was obtained from $P y 17 \mathrm{XL}-$ infected mouse tail and bled into saline sodium citrate, washed three times with PBS and adjusted to $10 \%$ hematocrit. Thin smears were prepared on microscope glass slides and quickly air-dried at RT. Sporozoite samples were obtained in PBS as described in section 2.1, seeded on twelve-well multi-test slides and quickly airdried at RT. The prepared slides were then stored at $-80^{\circ} \mathrm{C}$ with desiccants until use. The smears were thawed, fixed in $4 \%$ paraformaldehyde containing $0.0075 \%$ glutaraldehyde [25] (Nacalai Tesque) in PBS at RT for 15 
min, rinsed with $50 \mathrm{mM}$ glycine (Wako) in PBS. Sporozoites were permeabilized with $0.05 \%$ Triton $\mathrm{X}-100$ in PBS for $5 \mathrm{~min}$. Samples were then blocked with PBS containing $10 \%$ normal goat serum (Invitrogen) at $37^{\circ} \mathrm{C}$ for $30 \mathrm{~min}$. Next, samples were singly or doublyimmunostained with primary antibodies using purified rabbit anti-PyRON5 (final $2 \mu \mathrm{g} / \mathrm{mL}$ ), mouse monoclonal anti-PyRON4 (rhoptry neck marker, final $2 \mu \mathrm{g} / \mathrm{mL}$ ), crude mouse anti-PyRON2 serum (rhoptry neck marker, final 1:500), mouse monoclonal anti-PyRhopH3 (rhoptry body marker, final $2 \mu \mathrm{g} / \mathrm{mL}$ ), or crude mouse anti-PyAMA1 (micromene marker, final $1: 500$ ) at $37^{\circ} \mathrm{C}$ for $1 \mathrm{~h}$. Normal mouse or rabbit IgG (Millipore) or normal mouse serum was used as negative control. This was followed by 3 washes with PBS then incubation with Alexa Fluor ${ }^{\circledR} 488$ goat anti-mouse and/or Alexa Fluor ${ }^{\circledR} 594$ goat anti-rabbit antibodies (Invitrogen; final 500 times dilution) in blocking solution at $37^{\circ} \mathrm{C}$ for $30 \mathrm{~min}$. Parasite nuclei were stained with 4', 6-diamidino-2-phenylindole (DAPI; Invitrogen, final $0.2 \mu \mathrm{g} / \mathrm{mL}$ ). The stained samples were mounted with ProLong ${ }^{\circledR}$ Gold antifade reagent (Invitrogen) and cover slips onto microscope slides. Differential interference contrast (DIC) and fluorescence images were obtained using a fluorescence microscope (ECLIPSE 80i; Nikon, Japan) with a Plan Fluor 100x/1.30 oil immersion lens and a charge-coupled device camera (VB-7010; KEYENCE, Japan). Images were processed using Adobe Photoshop CS (Adobe Systems Inc., San José, CA).

\subsection{Immunoelectron microscopy (IEM)}

Schizont and sporozoite samples were fixed in a mixture of $4 \%$ paraformaldehyde containing $0.1 \%$ glutaraldehyde in $0.1 \mathrm{M}$ phosphate buffer, $\mathrm{pH} 7.4$, at $4^{\circ} \mathrm{C}$ for $15 \mathrm{~min}$. Fixed specimens were washed with $0.1 \mathrm{M}$ phosphate buffer, dehydrated with ethanol series $(30 \%$, $50 \%, 70 \%$, then $95 \%$ ), each time at $4^{\circ} \mathrm{C}$ for $5 \mathrm{~min}$, and embedded in LR White resin (London Resin Company, UK) as previously described [26]. Thin sections prepared by ultramicrotome (Reichert-Jung, Austria) were blocked in PBS containing 5\% non-fat milk (Becton, Dickinson and Company, USA) and $0.0001 \%$ Tween 20 (PBS-MT; Wako) at $37^{\circ} \mathrm{C}$ for $30 \mathrm{~min}$. Grids were then incubated with crude rabbit anti-PyRON5 serum in PBS-MT at $4^{\circ} \mathrm{C}$ overnight. After washing with PBS containing 5\% Blocking One buffer (Nacalai Tesque) and $0.0001 \%$ Tween 20 (PBS-BT), the grids were incubated at $37^{\circ} \mathrm{C}$ for $1 \mathrm{~h}$ with goat anti-rabbit IgG conjugated to $15 \mathrm{~nm}$ gold particles (EY Laboratories, San Mateo, CA) in PBS-MT, rinsed with PBS-BT, and fixed at RT in $0.5 \% \mathrm{OsO}_{4}$ (Nacalai Tesque) for $5 \mathrm{~min}$ to stabilize the gold. The grids were next rinsed with distilled water, dried, and stained with uranyl acetate and lead citrate. Samples were examined at $80 \mathrm{kV}$ under a transmission electron microscope (JEM-1230; JEOL Ltd., Tokyo, Japan). Final processing of the images was performed using Adobe Photoshop CS.

\section{Results}

\subsection{Determination of pyron5 gene and PyRON5 protein} primary structure

At the time of this study, the PY02282 gene (currently PY17X_0713300) had been identified as an ortholog of
PfRON5 (PF3D7 0817700, previously MAL8P1.73) in the Plasmodium database PlasmoDB [27]. We thus designated PY02282 as pyron5. The sequence of this gene was not completed. Nucleotide alignment using ClustalW between pyron 5 and pfron 5 open reading frames (ORFs) found the 3' side of the pyron5 missing. A BLASTN search was performed against $P$. berghei, using an orthologous pyron5 sequence as a query, and the complete gDNA sequence of pbron5 (PBANKA_071310) was identified. To complete and verify the exon-intron structure, DNA fragments covering the entire ORF were PCR-amplified from Py17XL cDNA. Fragments representing the 5' part and the middle part were amplified with PyRON5.F2-5U and PyRON5.R3, and PyRON5.F0 and PyRON5.R2 (Table 1). The pyron5 3' end was amplified using a forward primer based on the PY02282 3' end (PyRON5.F9) and reverse primers based on pbron5 3', end; PbRON5.R4 (based on the pbron5 3' ORF end) and PbRON5.R2 (based on the pbron5 3' UTR).

The full length ORF of pyron 5 was found to be 3,447 bp, distributed among 31 exons and encoding a protein (PyRON5) of 1,148 amino acid residues. The pyron5 cDNA sequence obtained from $P y 17 \mathrm{XL}$ completely matched the in silico prediction of the pyron5 cDNA sequence of the $P y 17 \mathrm{X}$ isolate described in PlasmoDB.

Multiple alignments of RON5 amino acid sequences were obtained using PyRON5, PfRON5 (ADV19050), TgRON5 (ACY08774), and the RON5 homolog of Theileria equi (BEWA_043030, designated as TeRON5 in this manuscript) [28], which was identified by TBLASTN search against EuPathDB transcripts database using $P y$ RON5 amino acid sequence as a query (Fig. S1) [29]. TgRON5 was proposed to be cleaved into 3 products; pro, RON5N, and RON5C domains at a sequence motif of "SFVE" or "SFVQ" by subtilisin-like protease, TgSUB2 [2, $30,31]$. However this motif does not exist in the other 3 RON5 sequences. SignalP 4.1 predicted a putative endoplasmic reticulum (ER) transport signal peptide at aa $1-20$ at the PyRON5 N-terminus (Fig. 1) similar to TgRON5, PfRON5 [5, 32] and TeRON5, suggesting that RON5 proteins are targeted to the secretory pathway. TM domains were not predicted by Phobius and OCTOPUS for $P y$ RON5. Amino acid alignment revealed that PyRON5, PfRON5, TgRON5, and TeRON5 had 5 conserved cysteine residues, 3 (at aa 341, 702, and 773) in the region corresponding to $T g R O N 5 N$ and 2 (at aa 804 and 858 ) in the region corresponding to $T g R O N 5 C$, which might play important roles for the protein conformation and function (Fig. 1 and Fig. S1). Other 4 cysteine residues of PyRON5 (at aa 262, 345, 713, and 733) were found to be conserved with PfRON5 (Fig. 1 and Fig. S1). There was a hydrophobic region at aa 553-577 in PyRON5 that was conserved among 4 RON5 amino acid sequences and not predicted to be a TM domain (Fig. 1, S1, and S4).

\subsection{PyRON5 was detected in the water-insoluble fractions}

Anti-PyRON5 detected multiple bands of various sizes from $\mathrm{FT}, \mathrm{Tx}$, and SDS fractions (Fig. 2A). Among 5 strongly reacted bands of these $(\sim 131-, \sim 87-, \sim 33-, \sim 31-$, and $\sim 26 \mathrm{kDa}$; Fig. $2 \mathrm{~A}$ arrowheads) seen in the FT fraction, the largest $\sim 131-\mathrm{kDa}$ band was about the same size as the calculated full length protein after excluding the putative signal peptide $(129 \mathrm{kDa})$ based on its amino acid sequence. The band around $131 \mathrm{kDa}$ in the water-soluble fraction 
was detected even after centrifugation at $>130,000 \times \mathrm{g}$ for $60 \mathrm{~min}$ (Fig. 2B arrowhead). Because these bands were reproducibly observed, we consider that the $\sim 87-, \sim 33-$, $\sim 31-$, and $\sim 26 \mathrm{kDa}$ bands are physiologically processed products. The $\sim 131-\mathrm{kDa}$ band was also detected in the Tx and SDS fractions. The $\sim 87-\mathrm{kDa}$ band was more prominent in the Tx fraction than in the FT fraction, and much reduced in the SDS fraction. The bands at $\sim 33-, \sim 31-$, and $\sim 26 \mathrm{kDa}$ were dramatically reduced in intensity in the Tx and SDS fractions, suggesting that these products do not associate with the membrane. No bands were detected with normal rabbit IgG.

\subsection{Complex formation of PyRON5 with PyRON4, $P y R O N 2$, and PyAMA1}

The reactivity of mouse anti-PyRON2 and rabbit antiPyAMA1 were confirmed with $P y 17 \mathrm{XL}$ late schizont extract by Western blotting (Fig. S2). Immunostaining was performed for proteins immunoprecipitated with antibodies against these proteins (Fig. 3). PyRON4 ( 140kDa), $P y$ RON2 (187-kDa processed form), and PyAMA1 $(60 \mathrm{kDa})$ were detected in the fraction immunoprecipitated with anti-PyRON5. In the reciprocal experiments, $P y$ RON5, a high intensity $\sim 131-\mathrm{kDa}$ band, was detected in the fractions immunoprecipitated with anti-PyRON4, anti$P y$ RON2, and, less intensely, with anti-PyAMA1. $P y$ RON5 $\sim 87-\mathrm{kDa}$ band (arrowhead in Fig. 3) was not detected even in the precipitate with anti-PyRON5, suggesting that the epitope region in the $\sim 87-\mathrm{kDa} P y$ RON5 product is not recognized by immunoprecipitation with this anti-PyRON5 antibody. In the precipitates with anti$P y$ RON2, anti-PyRON4, and anti-PyAMA1, all of these proteins were detected, except $P y$ RON2 in the precipitate with anti-PyAMA1, which is consistent with previous reports that failed or had difficulty to detect PfRON2 in precipitate with anti-PfAMA1 $[3,4]$. The band detected at $\sim 100 \mathrm{kDa}$ by anti-PyRON5 antibody was seen only in precipitates with rabbit control and anti-PyRON5, not in the Tx extract input, indicating that this is unrelated to RON5. Mouse and Rabbit control antibodies did not immunoprecipitate $P y \mathrm{RON} 2$ or $P y$ AMA1, but a faint $P y$ RON4 band was detected, which served as a background signal for RON4. This specific coimmunoprecipitation indicates complex formation among PyRON5, PyRON4, PyRON2, and PyAMA1.

\subsection{PyRON5 located at the rhoptry neck of the merozoite}

The localization of PyRON5 at the merozoite stage was evaluated by co-staining $P y$ RON5 with the known rhoptry neck markers, $P y$ RON4 and $P y$ RON2, the microneme marker, PyAMA1, and the rhoptry body marker, $P y$ RhopH3. In a segmented schizont, $P y$ RON5 antibody produced a punctate pattern of fluorescence and each signal was located at one end of each merozoite. $P y$ RON5 signals overlapped with PyRON4 (Fig. 4A, top panels) but only partly overlapped with $P y$ RON2, whose signal was detected in a wider area than PyRON5 (Fig. 4B, top panels). $P y$ RON5 signals also partly overlapped with PyAMA1 (Fig. 4C) or PyRhopH3 (Fig. 4D) signals. Negative controls did not produce any signal, indicating specific labeling of each antibody and no crosstalk between fluorescence channels (Fig. 4A and 4B, middle and bottom panels). To identify the precise location of
PyRON5, we performed IEM. Because purified anti$P y$ RON5 did not react with specimen for IEM, we used crude rabbit anti-PyRON5 serum. IEM precisely localized $P y$ RON5 at the neck portion of the rhoptries in the segmented schizont (Fig. 5), but preimmune rabbit serum did not react (Fig. S3B). Positive IFA image with this crude anti-PyRON5 serum and negative image with preimmune serum is provided in figure S3A. Thus $P y$ RON5 was identified as a rhoptry neck protein.

\subsection{PyRON5 is expressed and localized in the rhoptry of sporozoite derived from both oocysts and salivary glands}

Because P. berghei AMA1 and RON2 were previously shown to be expressed not only in merozoites but also sporozoites [15], we evaluated the expression of PyRON5 in sporozoites. Western blot analysis detected 3 bands at $\sim 99, \sim 64$, and $\sim 28 \mathrm{kDa}$ in the extract of oocyst-derived sporozoites (Fig. 6). The $\sim 99-\mathrm{kDa}$ band was found in the Tx fraction only and not detected in the schizont extract. The $\sim 64-\mathrm{kDa}$ band was detected in all FT, TX and SDS fractions and was similar to the broad faint band(s) around $\sim 64 \mathrm{kDa}$ in the schizont extract (arrow in Fig. 2A), which was detected in FT and Tx fractions, but not in the SDS fraction. The $\sim 28-\mathrm{kDa}$ band was strongly detected in water-soluble FT fraction, but only weakly in waterinsoluble fractions, which was similar to the weak $\sim 28$ $\mathrm{kDa}$ band detected in schizont extract in size and the extraction pattern. The bands at $\sim 131 \mathrm{kDa}$ and $\sim 87 \mathrm{kDa}$ detected in the schizont extract were not detected in the sporozoite extract.

IFA revealed that $P y$ RON5 was expressed in both types of sporozoites, one derived from oocyst and the other derived from salivary glands. Signals colocalized with PyRON4 at the apical end of the sporozoites, extending from the mid portion anterior to the nucleus to the apical tip of the sporozoite (Fig. 7). The signals were usually continuous, but occasionally, additional dot-like signals were observed towards the nucleus (Fig. 7 white arrowhead). IEM also revealed precise localization of $P y$ RON5 at the rhoptries in oocyst stage sporozoites (Fig. 8).

\section{Discussion}

In this study, we experimentally determined the ORF of RON5 in the rodent malaria parasite P. yoelii 17XL. Using $P$. yoelii, we showed that RON5 associates with the known MJ complex component proteins, RON2, RON4, and AMA1 in malaria parasites as it does in T. gondii [2]. Although TM prediction algorithms did not predict clear transmembrane region in PyRON5, a portion of RON5 was not extracted by repeated freeze-thaw procedure, suggesting that RON5 associates with membrane. We think that RON5 probably associates indirectly with membranes through interaction with a membrane-anchored protein in MJ complex proteins. RON5 is not only expressed in the merozoite, but also in the sporozoite.

The antibody that recognizes the N-terminal aa 36-53 of $P y$ RON5 labeled mature rhoptries both by IFA and IEM and detected $\sim 131-\mathrm{kDa} P y \mathrm{RON} 5$ product in the precipitates with anti-PyRON2, anti-PyRON4, or anti$P y A M A 1$ antibodies in schizont extracts, suggesting that the full length of RON5 is able to associate with MJ components. This is different from $T$. gondii, in which the 
antibody recognizing the pro-domain of $T g \mathrm{RON}$ only detected the pro-rhoptry compartment and did not detect mature rhoptries, and thus did not detect protein in the complex [33]. The homology of the N-terminal side of RON5 among different parasites is not high and reliable alignments could not be obtained. Also both PfRON5 and PyRON5 do not have the cleavage sites ("SFVE" or "SFVQ") present in TgRON5 [33]. Thus, it is impossible to predict the "pro-domain" in PyRON5, if it exists at all, by comparison with $T g R O N 5$. Nonetheless, the complex formation of the $\sim 131-\mathrm{kDa} P y$ RON5 product with other MJ complex components suggests that cleavage to form the $26-33 \mathrm{kDa}$ products may not be a prerequisite for complex formation. Alternatively, association of the $\sim 131-$ kDa $P y$ RON5 may be an artifact during the extraction procedure and only processed products that do not contain the N-terminal 26-kDa and $\sim 33-\mathrm{kDa}$ region may associate with other components at MJ during cell invasion. For example, the interaction between AMA1 and RONs detected in the schizont extracts is likely artificially achieved as AMA1 and RONs localized in the different compartments at schizont stage. Such artificial interaction may occur in $T$. gondii extract, for example, when $T$. gondii extract was immunoprecipitated with anti-TgRON4, a faint $\sim 175-\mathrm{kDa}$ band that appears to be a full-length $T g$ RON5 was also co-precipitated in addition to a $\sim 100$ $\mathrm{kDa} T g R O N 5 \mathrm{~N}$ band [2]. To clarify this point, future experiments are required using antibodies that react with the central and C-terminal parts of PyRON5.

Our anti-PyRON5 antibody detected a band around $\sim 87-\mathrm{kDa}$ in the P. yoelii schizont extract. Based on the molecular weight of $87 \mathrm{kDa}$, the $87-\mathrm{kDa}$ PyRON5 product is expected to contain an interspecies-conserved hydrophobic region at aa 553-577 in PyRON5 described above (Fig. S4). The reduction in intensity of the $\sim 87-\mathrm{kDa}$ band along with the presence of the $131-\mathrm{kDa}$ band in the Tx-insoluble SDS fraction suggests that the C-terminal is involved in PyRON5 association with detergent-resistant membrane or the removal of the $\mathrm{C}$-terminal part may prevent the association of the $\sim 87-\mathrm{kDa}$ product with detergent-resistant membrane.

The amino acid sequence alignment of PyRON5 and TgRON5 suggests that the cleavage event that yields $\sim 87$ kDa $P y$ RON5 product in $P$. yoelii schizont appears to be similar to that between RON5N and RON5C of TgRON5 (Fig. S4) [33]. However, the $\sim 87-\mathrm{kDa}$ product was not detected in sporozoites, whereas a $\sim 99-\mathrm{kDa}$ product was detected. Based on the expected cleavage site, the $\sim 87-\mathrm{kDa}$ product probably does not contain two interspeciesconserved cysteine residues at aa 804 and 858, as in $T g R O N 5$, but the $\sim 99-\mathrm{kDa}$ product may contain both of these cysteine residues. In $T$. gondii, the processing of $T g$ RON5N and $T g$ RON5C was dispensable without significant effect on function and rhoptry trafficking [33]. Thus this alternative cleavage may not affect protein function and may be due to differentially expressed RON5 maturases in merozoite and sporozoite.

Robust TM prediction algorithms, Phobius and OCTOPUS, that use statistical models trained with a set of known transmembrane regions did not predict any TM regions in $P y \mathrm{RON} 5$. Thus, the existence of $P y \mathrm{RON} 5$ in the water-insoluble $\mathrm{Tx}$ and $\mathrm{SDS}$ fractions suggests that PyRON5 has domain(s) that associate(s) with membrane(s) indirectly. RON2 has undisputed TM domain(s) and thus detection of PyRON5 in Tx and SDS fractions could be due to its association with PyRON2. Because the $\sim 100-\mathrm{kDa} T g R O N 5 \mathrm{~N}$ product is the main component in the MJ complex in $T$. gondii, the homologous central region of PyRON5 may be responsible for the MJ complex formation. In this regard, it should be noted that the interspecies-conserved hydrophobic region at aa 553-577 is located in this central region and the Cterminal from this hydrophobic region is relatively conserved among species.

In previous studies, AMA1, RON2, and RON4 were identified in sporozoites in addition to the merozoites [15, 16]. In this study, we show that RON5 is also expressed in both merozoites and sporozoites. Thus all four components forming the MJ complex are conserved between merozoite and sporozoite, suggesting that these proteins play roles in invasion not only of erythrocytes but also of mosquito salivary glands and/or hepatocytes..

\section{Acknowledgments}

We are grateful to T. Yoshimoto, K. Ito, and M. Kaewthamasorn for their valuable comments during experiments, T. Yanagi for support in maintaining rodent malaria parasites, R. Culleton for production of sporozoites, and D. Narum for anti-PyRON4 monoclonal antibody. This work was supported in part by Grants-inAids for Scientific Research 22390079 and for Scientific Research on Innovative Areas 23117008 (O.K.), and the Global COE Program, Nagasaki University, from the Ministry of Education, Culture, Sports, Science and Technology of Japan. J.K.M. is a recipient of MEXT PhD scholarship, Japan. All experiments conducted in this study were approved by the animal care and use committee, Nagasaki University.

\section{References}

[1] Baum J, Gilberger TW, Frischknecht F, Meissner M. Host-cell invasion by malaria parasites: insights from Plasmodium and Toxoplasma. Trends Parasitol 2008;24:557-63.

[2] Besteiro S, Michelin A, Poncet J, Dubremetz JF, Lebrun M. Export of a Toxoplasma gondii Rhoptry neck Protein complex at the host cell membrane to form the moving junction during invasion. PLoS Pathog 2009;5:e1000309.

[3] Alexander DL, Arastu-Kapur S, Dubremetz JF, Boothroyd JC. Plasmodium falciparum AMA1 binds a rhoptry neck protein homologous to TgRON4, a component of the moving junction in Toxoplasma gondii. Eukaryot Cell 2006;5:116973.

[4] Cao J, Kaneko O, Thongkukiatkul A, Tachibana M, Otsuki H, Gao Q, et al. Rhoptry neck protein RON2 forms a complex with microneme protein AMA1 in Plasmodium falciparum merozoites . Parasitol Int 2009;58:29-35.

[5] Lebrun M, Michelin A, El Hajj H, Poncet J, Bradley PJ, Vial H, Dubremetz JF. The rhoptry neck protein RON4 re-localises at the moving junction during Toxoplasma gondii invasion. Cell Microbiol 2005;7:1823-33.

[6] Chesne-Seck ML, Pizarro JC, Vulliez-Le, Normand B, Collins CR, Blackman MJ, B.W. Faber, et al. Structural comparison of apical membrane antigen 1 orthologues and paralogues 
in apicomplexan parasites. Mol Biochem Parasitol 2005;144:55-67.

[7] Alexander DL, Mital J, Ward GE, Bradley P, Boothroyd JC. Identification of the moving junction complex of Toxoplasma gondii: a collaboration between distinct secretory organelles. PLoS Pathog 2005;1:e17.

[8] Riglar DT, Richard DW, Wilson, Boyle MJ, Dekiwadia CL. Turnbull F, et al. Super-resolution dissection of coordinated events during malaria parasite invasion of the human erythrocyte. Cell Host Microbe 2011;9:9-20.

[9] Lamarque M, Besteiro S, Papoin J, Roques M, Vulliez-Le Normand B, J. Morlon-Guyot, et al. The RON2-AMA1 interaction is a critical step in moving junction-dependent invasion by apicomplexan parasites. PLoS Pathog 2011;7:e1001276.

[10] Srinivasan P, Beatty WL, Diouf A, Herrera R, Ambroggio X, Moch JK, et al. Binding of Plasmodium merozoite proteins RON2 and AMA1 triggers commitment to invasion. Proc Natl Acad Sci USA 2011;108:13275-80.

[11] Vanderberg JP, Frevert U. Intravital microscopy demonstrating antibody-mediated immobilisation of Plasmodium berghei sporozoites injected into skin by mosquitoes. Int J Parasitol 2004;34:991-6.

[12] Yahata K, Treeck M, Culleton R, Gilberger TW, Kaneko O. Time-lapse imaging of red blood cell invasion by the rodent malaria parasite Plasmodium yoelii. PLoS One 2012; 7:e50780.

[13] Frischknecht F, Baldacci P, Martin B, Zimmer C, Thiberge S, Olivo-Marin JC, et al. Imaging movement of malaria parasites during transmission by Anopheles mosquitoes. Cell Microbiol 2004;6:687-94.

[14] Lasonder E, Janse CJ, van Gemert GJ, Mair GR, Vermunt AM, Douradinha BG, et al. Proteomic profiling of Plasmodium sporozoite maturation identifies new proteins essential for parasite development and infectivity. PLoS Pathog 2008;4:e1000195.

[15] Tufet-Bayona M, Janse CJ, Khan SM, Waters AP, Sinden RE, Franke-Fayard B. Localisation and timing of expression of putative Plasmodium berghei rhoptry proteins in merozoites and sporozoites. Mol Biochem Parasitol 2009;166:22-31.

[16] Giovannini D, Späth S, Lacroix C, Perazzi A, Bargieri D, Lagal V, et al. Independent roles of apical membrane antigen 1 and rhoptry neck proteins during host cell invasion by apicomplexa. Cell Host Microbe 2011;10:591-602.

[17] Narum DL, Ogun SA, Thomas AW, Holder AA. Immunization with parasite-derived apical membrane antigen 1 or passive immunization with a specific monoclonal antibody protects BALB/c mice against lethal Plasmodium yoelii yoelii YM blood-stage infection. Infect Immun 2000;68:2899-906.

[18] Narum DL, Nguyen V, Zhang Y, Glen J, Shimp RL, Lambert L, et al. Identification and characterization of the Plasmodium yoelii PyP140/RON4 protein, an orthologue of Toxoplasma gondii RON4, whose cysteine-rich domain does not protect against lethal parasite challenge infection. Infect Immun 2008;76:487682.

[19] Edgar RC. MUSCLE: multiple sequence alignment with high accuracy and high throughput. Nucleic Acids Res 2004;32:1792-97.

[20] Petersen TN, Brunak S, von Heijne G, Nielsen H. SignalP 4.0: discriminating signal peptides from transmembrane regions. Nat Methods 2011;8:785-6.

[21] Käll L, Krogh A, Sonnhammer ELL. A combined transmembrane topology and signal peptide prediction method. J Mol Biol 2004;338:1027-36.

[22] Viklund $\mathrm{H}$ and Elofsson A. Improving topology prediction by two-track ANN-based preference scores and an extended topological grammar. Bioinformatics 2008;24:1662-8.

[23] Shirano M, Tsuboi T, Kaneko O, Tachibana M, Adams JH, Torii M. Conserved regions of the Plasmodium yoelii rhoptry protein RhopH3 revealed by comparison with the $P$. falciparum homologue. Mol Biochem Parasitol 2001;112:297-9.

[24] Kaneko O, Fidock DA, Schwartz OM, Miller LH. Disruption of the C-terminal region of EBA-175 in the Dd2/Nm clone of Plasmodium falciparum does not affect erythrocyte invasion. Mol Biochem Parasitol 2000;110:135-46.

[25] Tonkin CJ, van Dooren GG, Spurck TP, Struck NS, Good RT, Handman E, et al. Localization of organellar proteins in Plasmodium falciparum using a novel set of transfection vectors and a new immunofluorescence fixation method. Mol Biochem Parasitol 2004;137:13-21.

[26] Aikawa M, Atkinson CT. Immunoelectron microscopy of parasites. Adv Parasitol 1990; 29:151-214.

[27] Bahl A, Brunk B, Crabtree J, Fraunholz MJ, Gajria B, Grant GR, et al. PlasmoDB: the Plasmodium genome resource. A database integrating experimental and computational data. Nucleic Acids Res 2003;31:212-5.

[28] Kappmeyer LS, Thiagarajan M, Herndon DR, Ramsay JD, Caler E, Djikeng A, et al. Comparative genomic analysis and phylogenetic position of Theileria equi. BMC Genomics 2012;13:603.

[29] Aurrecoechea C, Heiges M, Wang H, Wang Z, Fischer S, Rhodes P. et al. ApiDB: integrated resources for the apicomplexan bioinformatics resource center. Nucleic Acids Res 2007;35:D427-30.

[30] Straub KW, Cheng SJ, Sohn CS, Bradley PJ. Novel components of the Apicomplexan moving junction reveal conserved and coccidia-restricted elements. Cell Microbiol 2009;11:590-603.

[31] Miller SA, Thathy V, Ajioka JW, Blackman MJ, Kim K. TgSUB2 is a Toxoplasma gondii rhoptry organelle processing proteinase. Mol Microbiol 2003;49:883-94.

[32] Curtidor H, Patiño LC, Arévalo-Pinzón G, Patarroyo ME, Patarroyo MA. Identification of the Plasmodium falciparum rhoptry neck protein 5 (PfRON5). Gene 2011;474 :22-8. 
Beck JR, Chen AL, Kim EW, Bradley PJ. RON5 is critical for organization and function of the Toxoplasma moving junction complex. PLoS Pathog 2014;10:e1004025.

\section{Figures}

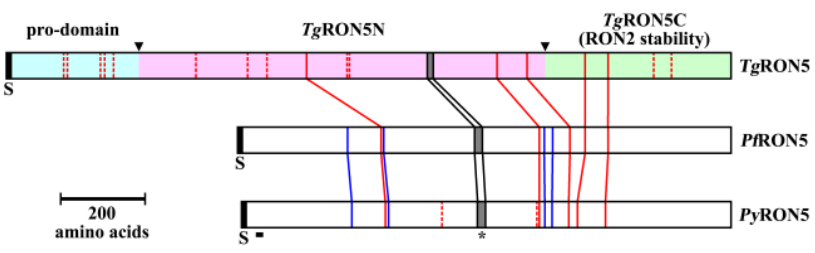

Figure 1. Primary protein structure of RON5 in Plasmodium yoelii, Plasmodium falciparum, and Toxoplasma gondii. The solid red lines indicate five conserved cysteine residues among PyRON5, PfRON5 and TgRON5 based on the multiple sequence alignment including Theileria equi RON5 homolog (Fig. S1). The dotted red lines indicate the unique cysteine residues. The solid blue lines indicate the additional four conserved cysteine residues between PyRON5 and PfRON5, making nine-conserved cysteine residues between the two. The short black bold horizontal line under PyRON5 indicates the relative position of a peptide sequence used to generate and purify anti-PyRON5 antiserum. $\mathrm{S}$ indicates putative signal peptide. Gray boxes, marked with asterisk, indicate a relatively conserved hydrophobic region among RON5 sequences consisting of multiple conserved amino acids (Fig. S1). The black arrowheads indicate TgRON5 cleavage sites. Pro-domain, RON5N, and RON5C regions in $T$. gondii RON5 are indicated with cyan, pink, and green colors, respectively.
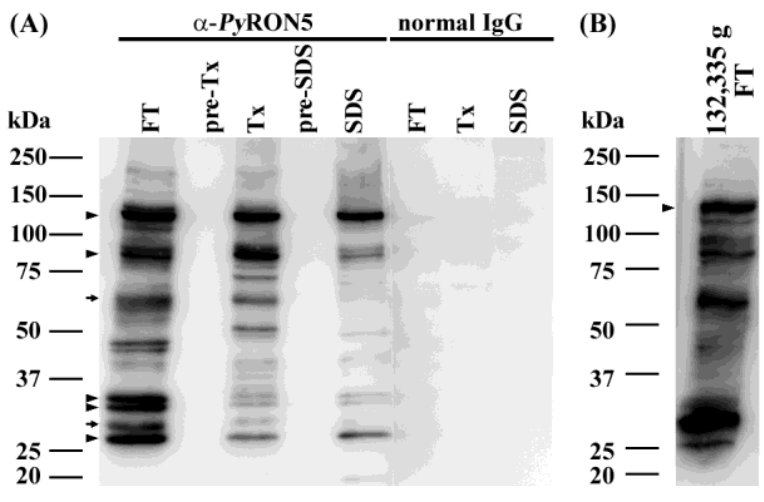

Figure 2. Western blot of RON5 in Plasmodium yoelii blood stage parasites. (A) Equal amount of endogenous proteins sequentially extracted from schizont rich parasite pellet by freeze/thaw (FT), 1\% Triton X-100 (Tx), and 2\% sodium dodecyl sulphate (SDS) were subjected to Western blot analysis using purified rabbit anti-PyRON5 ( $\alpha-$ PyRON5) antibody. Pre-Tx or pre-SDS indicates FT or Tx extraction before proceeding to the next extraction step, respectively. Rabbit normal IgG was used as a negative control. Arrowheads indicate the major 5 bands detected in FT fraction around 131, 87, 33, 31, and $26 \mathrm{kDa}$. Arrows indicate the minor bands around 64 and $28 \mathrm{kDa}$ discussed in the manuscript. (B) The FT fraction from schizont extracts was also obtained by centrifugation at $132,335 \times \mathrm{g}$ for $60 \mathrm{~min}$ and subjected to Western blot analysis using purified rabbit anti-PyRON5. An arrowhead indicates a band around $131 \mathrm{kDa}$.

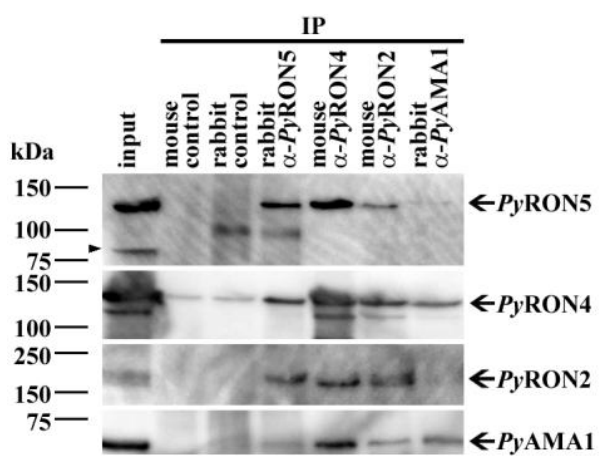

Figure 3. Complex formation of RON5 with RON4, RON2, and AMA1 in Plasmodium yoelii. Protein from late schizonts was extracted with Triton X-100 (input) and immunoprecipitation (IP) was performed with purified rabbit anti-PyRON5 ( $\alpha$-PyRON5) antibody, mouse anti$P y$ RON4 ( $\alpha-P y$ RON4) monoclonal antibody, crude mouse anti-PyRON2 ( $\alpha-P y$ RON2) serum, purified rabbit antiPyAMA1 antibody, mouse antibodies (mouse control) or rabbit control serum (rabbit control), then detected with a panel of antibodies described above. Arrows indicate the expected bands for each protein. Arrowhead indicates $\sim 87-$ $\mathrm{kDa}$ product of $P y \mathrm{RON} 5$.

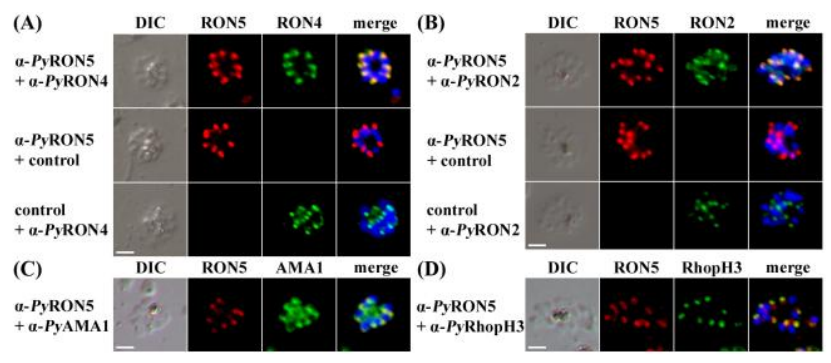

Figure 4. Immunofluorescence assay of RON5 with rhoptry neck (RON4 and RON2), microneme (AMA1), and rhoptry body (RhopH3) marker proteins in Plasmodium yoelii schizont stage merozoites. Schizontinfected erythrocytes were dual-labeled with purified rabbit anti-PyRON5 (red) and (A) mouse anti-PyRON4 (green), (B) mouse anti-PyRON2 (green), (C) mouse antiPyAMA1 (green), or (D) mouse anti-PyRhopH3 (green). Control stainings were done with rabbit preimmune sera or normal mouse IgG to exclude the possible false signals from crosstalk between fluorescence channels. The differential interference contrast (DIC) and merged images with nuclei stained with DAPI (blue) are shown. Scale bar represents $2 \mu \mathrm{m}$. 


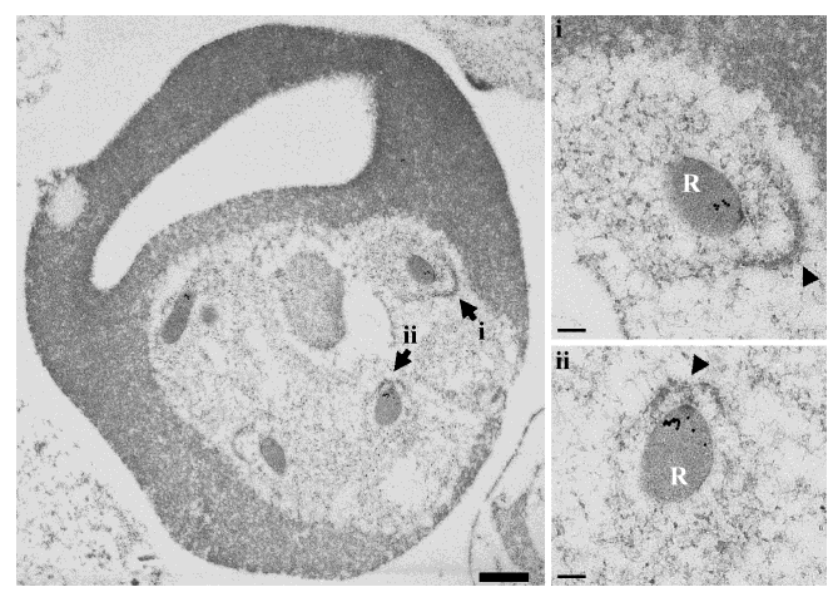

Figure 5. Localization of RON5 in Plasmodium yoelii schizont stage merozoites by immunoelectron microscopy. Longitudinally sectioned merozoites in schizont-infected erythrocytes were stained with rabbit anti-PyRON5 serum followed by secondary antibody conjugated with gold particles. Schizont stage daughter merozoites exhibiting rhoptries (R) are indicated in the cropped and enlarged images on the right side (i and ii). Arrowheads indicate the apical end of the merozoite. Thick and thin scale bars represent 0.5 and $0.2 \mu \mathrm{m}$, respectively. Control images stained with rabbit preimmune serum can be seen in Fig. S3B.

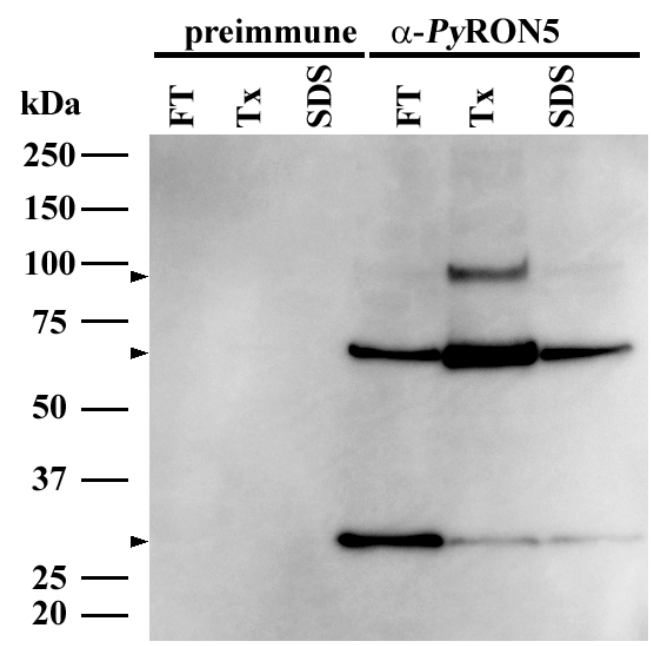

Figure 6. Western blot of RON5 in Plasmodium yoelii sporozoites. Equal amount of endogenous proteins sequentially extracted from oocyst-derived sporozoites by freeze/thaw (FT), 1\% Triton X-100 (Tx), and 2\% sodium dodecyl sulphate (SDS) were subjected to Western blot analysis using rabbit anti-PyRON5 ( $\alpha$-PyRON5) or preimmune sera. Arrowheads indicate 3 detected bands around 99, 67, and $28 \mathrm{kDa}$.

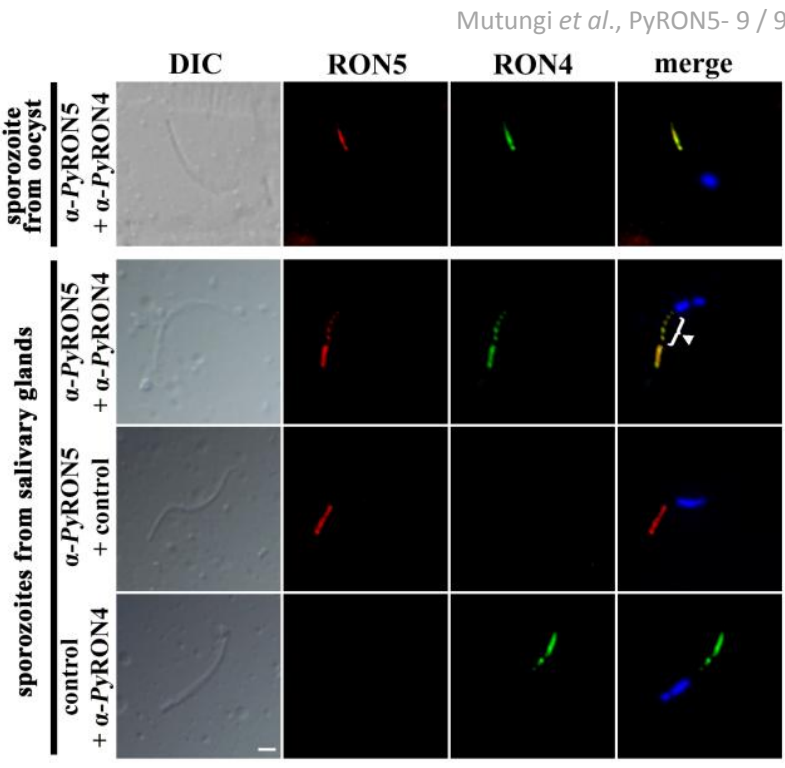

Figure 7. Immunofluorescence assay of RON5 with rhoptry neck marker protein, RON4, in Plasmodium yoelii sporozoites. Sporozoites obtained from ruptured mature oocyst (top panel) or from salivary glands of the mosquitoes (bottom 4 panels) were stained with purified rabbit anti-PyRON5 antibody and mouse monoclonal anti$P y$ RON4 antibody. Rabbit normal IgG and mouse normal IgG were used as negative controls. The differential interference contrast (DIC) and merged images with nuclei stained with DAPI (blue) are shown. Dot-like signals are indicated with white arrowhead and parenthesis. Scale bar represents $2 \mu \mathrm{m}$.

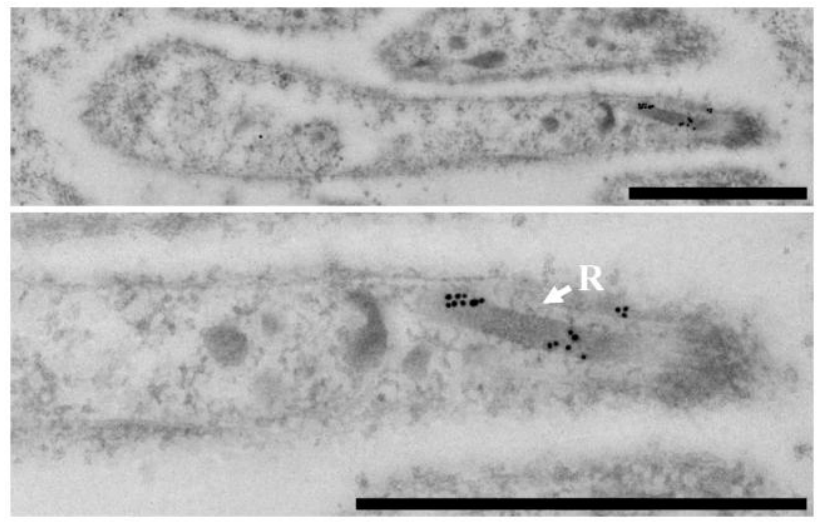

Figure 8. Localization of RON5 in Plasmodium yoelii sporozoites by immunoelectron microscopy. Sectioned mature oocyst stage sporozoites were labeled with rabbit anti-PyRON5 serum. Cropped and enlarged image is shown below. Scale bars represent $1 \mu \mathrm{m}$. Rhoptry (R) is indicated. Control image stained with rabbit preimmune serum can be seen in Fig. S3C. 
Table 1.

Primers used for PCR amplification and sequencing of pyron5.

Primers for PCR

PyRON5.F2-5U

PyRON5.R3

PyRON5.F0

PyRON5.R2

PyRON5.F9

PbRON5.R4

PbRON5.R2

Primers for sequencing

PyRON5.F1

PyRON5.F2

PyRON5.seqF0

PyRON5.seqF1

PyRON5.seqF2

PyRON5.R0

GAAAGCCATTTGCATTTATTTGTGTGTG

CATTTCGGTGATTGTTCTTAAATAGTGAA

ATGAAATTGAGTATACTATTTTATGTGG

GCATAGTATTCTTTAATATTGCTT

CATGGATACTTCTTGATAAATTGTCC

TTAAGGTATTCTTGTATGAACAATAATTTC

CAACCACATACATATATATATGTTTGC

PyRON5.R1

PyRON5.seqR0

PyRON5.seqR1

PyRON5.seqR2

GTGGGGTTTATAGATATGTGTT

GTAAACCAGCTATTGTTCCTAT

GCAACTATGTATTCTATAGATTC

GGTTATGGGATGAGATTCAGA

GACAGTGTTGTTACTGTAAAAG

CTCTTCTTTGATTAGTATCTTGAG

CATCATAATATTTACATCTTGTAG

CAATGGTGTTTTGTATATGTTTAG

CCATCTAAAAAGAAATCCAACAT

CTTGATTTGGATAATCCGATGT 


\title{
Supporting Online Material
}

\section{Expression and localisation of rhoptry neck protein 5 in merozoites and sporozoites of Plasmodium yoelii}

\author{
Joe Kimanthi Mutungi ${ }^{\mathrm{a}, \mathrm{b}}$, Kazuhide Yahata $^{\mathrm{a},{ }^{*}}$, Miako Sakaguchi ${ }^{\mathrm{c}}$ \\ and Osamu Kaneko, ${ }^{\text {a, }}$
}

${ }^{a}$ Department of Protozoology, Institute of Tropical Medicine (NEKKEN), Nagasaki University, Sakamoto, Nagasaki 852-8523, Japan.

${ }^{\mathrm{b}}$ Graduate School of Biomedical Sciences, Nagasaki University, Sakamoto, Nagasaki 852-8523, Japan.

${ }^{\mathrm{c}}$ Central Laboratory, Institute of Tropical Medicine. (NEKKEN), Nagasaki University, Sakamoto, Nagasaki 852-8523, Japan.

* Corresponding authors: Tel.: +81 95819 7838; fax: +81 95819 7805; E-mail address: kyahata@nagasaki-u.ac.jp or okaneko@nagasaki-u.ac.jp

\section{Supplementary Table and Figures}

Figure S1. Multiple sequence alignment of RON5 amino acid sequences. Amino acid sequences of Plasmodium yoelii RON5 (PyRON5, in this study), Plasmodium falciparum RON5 (PfRON5, ADV19051), Toxoplasma gondii RON5 (TgRON5, ACY08774) and Theileria equi RON5 homolog (BEWA_043030) were used to make a multiple sequence alignment using MUSCLE webware. Periods and colons indicate semi-conserved, and asterisks indicate conserved amino acid residues. $T g$ RON5 pro, $T g$ RON5N, and $T g R O N 5 C$ regions are indicated above the alignment with cyan, pink, and green, respectively. Cleavage sites of TgRON5 proposed or confirmed by Beck et al (2014) [1] are indicated with pink box. Signal peptide sequence predicted by SignalP4.1 (with sensitive Dcutoff values) are boxed with dark green and white letter. Cysteine residues conserved among at least 3 spp. are highlighted with white letter and red box and cysteine residues not conserved with white letter and black box. A hydrophobic region consisting of conserved amino acids among $4 \mathrm{spp}$. is masked with gray box.

Figure S2. Western blot of mouse anti-PyRON2 serum and rabbit anti-PyAMA1 antibody used in the study. Schizont extracts with $1 \%$ Triton X-100 was subjected to Western blot with crude mouse anti-PyRON2 serum, normal mouse serum, and purified rabbit anti-PyAMA1 antibody. Mouse anti- $P y$ RON2 serum reacted with $>250-\mathrm{kDa}$ consistent with the full length of $P y \mathrm{RON} 2$ (expected $\mathrm{MW}=252 \mathrm{kDa}$ after excluding signal peptide) and three other bands at $\sim 215, \sim 183 \mathrm{kDa}$, which were detected most strongly, and $82 \mathrm{kDa}$. No band was detected with control normal serum. Purified rabbit anti-PyAMA1 reacted with a single $60-\mathrm{kDa}$ band as expected [2]

Figure S3. Indirect immunofluorescence assay with rabbit anti-PyRON5 crude serum and its preimmune serum and immunoelectron microscopy images with rabbit preimmune serum for anti-PyRON5. (A) Schizont-infected erythrocytes were labeled with crude rabbit anti-PyRON5 (red) or its preimmune serum. The differential interference contrast (DIC) and merged images with 
nuclei stained with DAPI (blue) are shown. Scale bar represents $2 \mu \mathrm{m}$. (B) Plasmodium yoelii schizont stage merozoites and (C) sporozoites in oocyst are shown. Scale bars represent 0.2 and 1 $\mu \mathrm{m}$ for merozoite and sporozoite images, respectively. Arrows indicate rhoptry (R). No gold particle signals were detected.

Figure S4. Primary protein structure of RON5 in Plasmodium yoelii, Plasmodium falciparum, and Toxoplasma gondii and schematics of the expected processing of PyRON5. Top panel was explained in the legend of figure 1. In the bottom panel, the solid red lines, the solid blue lines, and the dotted red lines indicate conserved cysteine residues among PyRON5, PfRON5 and TgRON5, conserved cysteine residues between PyRON5 and PfRON5, and unique cysteine residues in $P y$ RON5, respectively. Gray boxes indicate a relatively conserved hydrophobic region among RON5 sequences consisting of multiple conserved amino acids. C-terminal from this hydrophobic region is more homologous than the $\mathrm{N}$-terminal from this region (Fig. S1). N-terminal region having no homology with $T g R O N 5$, a region having some homology with $T g R O N 5 N$, and that having clear homology with $T g R O N 5 \mathrm{C}$ are shown with yellow, pink, and green, respectively. Potential cleavage sites are indicated with waving lines.

\section{References}

[1] Beck JR, Chen AL, Kim EW, Bradley PJ. RON5 is critical for organization and function of the Toxoplasma moving junction complex. PLoS Pathog 2014;10:e1004025.

[2] Narum DL, Ogun SA, Thomas AW, Holder AA. Immunization with parasite-derived apical membrane antigen 1 or passive immunization with a specific monoclonal antibody protects $\mathrm{BALB} / \mathrm{c}$ mice against lethal Plasmodium yoelii yoelii YM blood-stage infection. Infect Immun 2000;68:2899-906. 
Table S1. Amino acid sequences of the peptides used for immunization

protein amino acid positions sequence

$\begin{array}{lll}\text { PyRON5 } & 36-53^{*} & \text { NNHRNDSKLEHSNKNTFD-C } \\ \text { PyRON5 } & 448-461 & \text { APFKFFRDPQDKSY-C } \\ \text { PyRON5 } & 1110-1138 & \text { C-IVNLKNATFTLNGILNFVIKAEKGNDN } \\ & & \\ P y \text { RON2 } & 1199-1213 & \text { C-NQNEYQRQNQIKNQN } \\ P y \text { RON2 } & 1929-1942 & \text { C-GKWQKERQQGRLKE } \\ P y \text { RON2 } & 2110-2122 & \text { C-NEIHNNTRGNYHY } \\ P y A M A 1 & 289-303 & \text { C-ESASDQPKQYERHLE } \\ P y A M A 1 & 336-352 & \text { C-KSHGKGYNWGNYDSKNN } \\ P y A M A 1 & 505-526^{*} & \text { C-GKKGENYDRMGQADDYGKSKSR }\end{array}$

Cysteine residue was added to the $\mathrm{N}$ - or C-terminus of each peptide to conjugate with KLH. Asterisks indicate peptides that gave the best antibody after affinity purification. 
Fig. S1. Multiple sequence alignment of RON5 amino acid sequences

\begin{tabular}{|c|c|}
\hline & TgRON5 pro-domain \\
\hline TgRON5 & MAEFTWRPLLMSLPKMI AFFHILLFSGALAAAAGSPAADLVASVQTVSNERKDLYARDTQPTARTGIDIGVSFTQQASGNARTFEIRQHGSGPP---RPAPRRAAAVADDI-------FGS \\
\hline \multirow{4}{*}{$\begin{array}{l}\text { BEWA_043030 } \\
\text { PyRON5 } \\
\text { PfRON5 }\end{array}$} & MI GSGSKS------RIFYYLI ILFLVINLKNVYRVYALSGEFDANGNGI TQNSLTSTT IPTTI INQEQSTDENKSESIDSVNKVPDLKSSDQLFP---KPDSQKNEVDQSGHKPIDQLPPS \\
\hline & 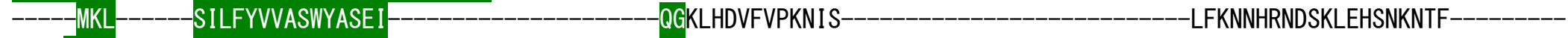 \\
\hline & 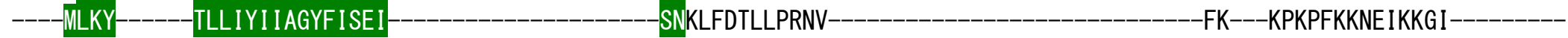 \\
\hline & . $\quad:: \quad: \quad:$ \\
\hline TgR0N5 & EDFSPPPMNVAGAPLRDMGVHFLECQATDGKIECTGQGAGARPPFFRGGVDPTEIHEIVQSRTVGPADYDEERPEQTPDYLSPTDVVTLQRFVSSANASNSPLLEDPVQVCLSRRKPTYT \\
\hline BEWA_043030 & DDYDEDDVRGIDEKSGE I SRYTLGVDQEWLRRENAAKVVGSKLP----------------ASTSVG---YNKRTHTLEPI-LSAQKTL----------GGVIQSLVDNESI--LRRKYSQK \\
\hline PyR0N5 & 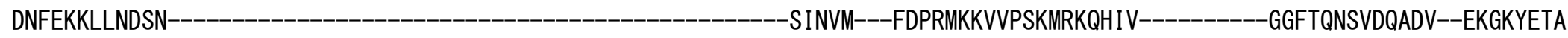 \\
\hline \multirow[t]{3}{*}{ PfRON5 } & 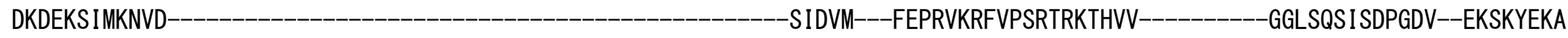 \\
\hline & $\begin{array}{llllllllllll}* & * & : & * & * & : & * & \ldots & * & \end{array}$ \\
\hline & proposed TgR0N5N \\
\hline TgR0N5 & CHLLHEFAATSVIVEESGNLVCEDKAPLTVAEKRKINDAVKAGRTPQATGGQSSRPPNPTVSPSKAGAAPQNAASRQAVSFVEQENSEASMPTANTEQASATTEDTKIASAATDSGDYGE \\
\hline BEWA_043030 & 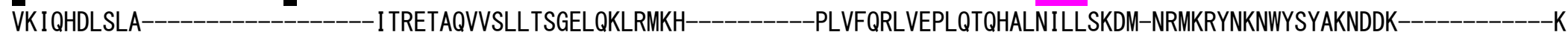 \\
\hline PyR0N5 & 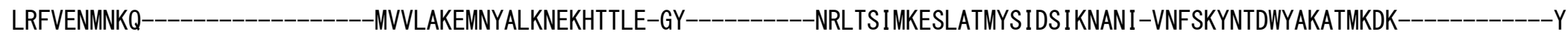 \\
\hline \multirow[t]{2}{*}{ PfR0N5 } & VRFFENIKNE--------------------M I NMSSK INKQLDSQDISSLN-NF-----------KRASEVLKESLATMHSLDI IRNDGS-VDFSKYTLDWYSKANMREK-------------Y \\
\hline & 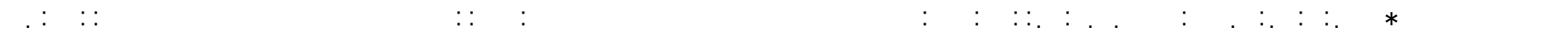 \\
\hline TgR0N5 & AAAGESAQEGDRPPPYNPD-ADEAGVPRAVQEAYEEARPLQ-EATIDKFKQDAAAAAEAADHFAQVSAFNAMQSALTKISAGYHLRAGAHVVLSACKRLVEAVAANPPGPGTVIPLEELR \\
\hline BEWA_043030 & 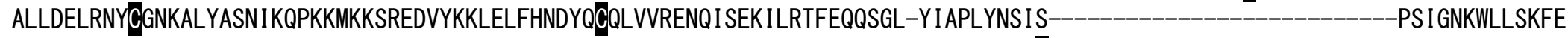 \\
\hline PyRON5 & DAAKHI QNTIDK--LFKPS-NKKKSVKEKK ID--ENIKQLETDLLLQRFVMDNSNVSMLLKKYEDSGDDKYMAPSYTDVC-------------------------NQLGHPILSYVFE \\
\hline \multirow[t]{2}{*}{ PfRON5 } & SIEKSIQK IMNK--LFKKARKKKKNMKKKK ID--ANIEQLEMDLLVQKF I TENLNASKLLKLYDDSAND-YVSPMHTDVC-. \\
\hline & 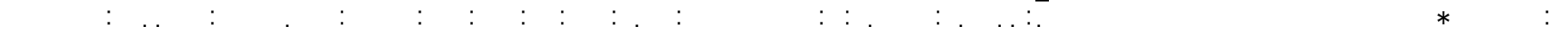 \\
\hline TgR0N5 & MQLVATLTQDFALAQAF IDYAIHIVHSAIETLTPQMVANALLELSGIEEL INHTARVKSRLAARGQDSPANVRKEIVQESFRQLKVELFQEIVTRVCELMDDPESFLETVPI IVGTTPTA \\
\hline BEWA_043030 & 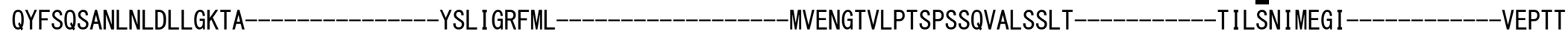 \\
\hline PyR0N5 & 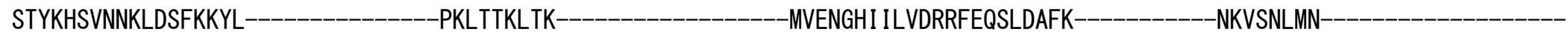 \\
\hline \multirow[t]{2}{*}{ PfR0N5 } & KAYKSS INHD I SYFQKYL-----------------PRLKYRI QN--------------------MI QKGTLLLLEKGLDDSLYTFK------------SK ISDIME--- \\
\hline & : $: \quad: \quad:$ \\
\hline
\end{tabular}


Fig. S1. Multiple sequence alignment of RON5 amino acid sequences (continued)

TgRON5

BEWA_043030

PyRON5

PfRON5

TgRON5

BEWA_043030

PyRON5

PfRON5

TgRON5

BEWA_043030

PyRON5

PfRON5

TgRON5

BEWA 043030

PyRON5

PfRON5

TgRON5

BEWA_043030

PyRON5

PfRON5

PLRTGGHLGADYI I IHLRNDLCDVTASDAQIFPSAPADEGLQGFPRHNLGERLVGWMDVMARTKTARKEVFKI IDFTKAKDVLLFASETGKARYATLQAPAAPAPSFYGI TTSGCRNVNDI FLGKKKYYGF---_---MNMCDVKCAEGLFKP--_-_--KSNFFYKELLNK--_-_-_-_--_QLEILKWVTAF-YNDDLLRIDTSAQKLLLEIMYRTTRDPGFLRSYKSVRLNIKSE -----KKVGF------_IDMCSKKCFDDTVE INYKLDKYDIKLSPQDTNQR-----------RGDLTRMVLYY-YKEI INHIEANAD IVL IMLLHLSSSA----KT IESGRLDIDIA

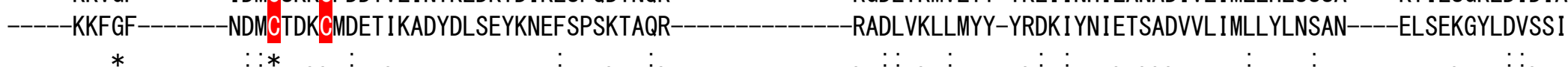

* $:: *$. . . . $\quad$.

MKSKFFDMYLHQSGFMKLQRHGNDNSRORFMHRVTLLQNDGILPQLPLEADYELM--ELNAAMOKNFVAANKSIFSRRAARHSKYGYLDLCDVACYOKIDRLHNDVMTNVFFSLDTTLMK FEDKDKSF IELDTPIPVPIKFQKLERVTKNTGQLQDVTDENKPTKSPIMGKFKIFMSRFLSGFRKQGSSVDNKALSSSIV---PYEVFK-TPKNIVASLDNLFLD-----TFYNIADYI--

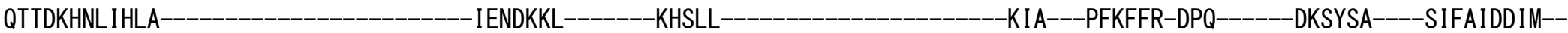

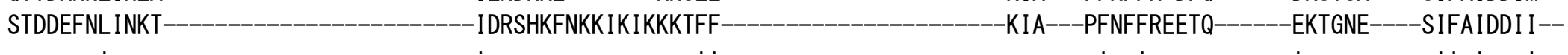
: $\therefore:$ :

IVAKVHRSYGIAKAFFQLGARQQHIADPNLGMWARRLFVHWASHNEVKQMQGQKVVKVNYENLRHGEFTLDTVRMRDALVRYTNMLKADPITRDLMSLVIHTWIHIRGVRNAAMGFKNSQ -----KSAA---_----EHENNVYYETLNTF----_----VLI QNIHGAIHSF---EDKKTKSLASS--KTLGAVFRWLNM---N--PREHLSHIPNKFL -----KTCSLSKKF----KNYDSLYEKTKGLW---_--_--DEI QNLYSASYGFVPSKKIKTQSF I TSK IRNVGFLFRWLNN---NEYTSSDVNFLGKNYS------KTSLLAKKS----QNYNSLYETTKDLW----------NQI QNMYSASYGFVQSKKIKTNKFVGSKIRNVGFLLRWFNY---NKTPSKNINFLVNNFS

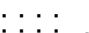

KLNESMNASAIGAVFAKLWYESD I SVVAPHQELKPFGAPLASMALQIGFFLHTVVEEYKMSLLEKAGAQIKSWFVGMFQKNKRRT-VPRTWKAVVAATNRAPKVNLKAYQGALLVIKTLA PFVSLSLQLTFFINTMIEQYESSFL----GNFSAAIKKIFTLGKSGA-NPKNYDDLINFSETDYLLRTKKADAVQRI INQT I PLVSISLQLVFFITTMIEQYESSFL----GNFSSALKKIFTLGKSGR-NPRNYNDLVNFSEVDYLLRTSKANNVQRI IMQI I

KMFRERFLYRFYMQGQGSKVDFNTPTLLIH---ALVASWMDPSLDRLELSSRT IPNAKKLFWYYVWVNENGPSNAATRIVLTGCKKYTF-LLPGVVRSVTSSTS-EVVEAGSNILKIDKI SKFKDLFLSKPA----------IPSPI I QY I TVFVGLWAKGASGDFSMSD INMDRVRKTFFLSYVSNGKSLADMATNI IMDNCKVGNHALHLGCI ISK ISS---------NNKCKQIYV KILKKKFFSGEY---------TPTLFAQYVSLFLSLWVFEGEKDI SMNNPNI SRFKKIFFLSFLVHNSGVVEKATEI I CKSCOKKTKKIVLGCIDDYGGKTKRKILGIFSKKCKPAIV RMLKKKFLSSSY----------TPTLLAQYMSIFLSLWVFEGENNI SLQNPNI SRFKK IFFL TYFVHEKGPVEKAVDI I YNKCRMKTDK IVLGCI IHDYGGREKKKLLGGL I SRKCKPTK I 
Fig. S1. Multiple sequence alignment of RON5 amino acid sequences (continued)

TgRON5

BEWA_043030

PyRON5

PfRON5

TgRON5

BEWA_043030

PyRON5

PfRON5

TgRON5

BEWA_043030

PyRON5

PfRON5

TgRON5

BEWA 043030

PyRON5

PfRON5

TgRON5

BEWA_043030

PyRON5

PfRON5

I ILKRSSLEAYMNHLQATYDDPLTIVQVALDLAARCEGYSAAKDQPAQAMRGPARVRRATGESTTFT IRGGGVQGGTMSFVEAEADDERKEDSEDNTVDLSEQDQDSSFVQLKKLFNRRGS SLKSNKLKKVLKMLEATFHDPLDI I RLASDTARRCLAQ

PIEKKSVRKILKVLMTTLSDPLDILKIAVDLSTRCKYY-

SIRKRSIRKILNKLMSSLNDPVDILRIAVDTATRCDHF-

SAAGQAVQTDAQPLPKAVQTDAQPLPKVRRGGPDVDAS----AVILGSRFMLDLWCSKYREMLVEKL--SGISTKDATVMQQEI SKVFSAVSSIKIAIPDYKDL-WDFSLRCDWMDGYPD -------KKQPRPRQSMKRSKPYKYFPLAKKT I KVPHYMDRLMLHSEFSHRIHCYKAQKRLVKEMIKSLSSAVSEDSARKI I STVFSSYRSIE--IKQVGMANSSYRL ICPFMYDAPE

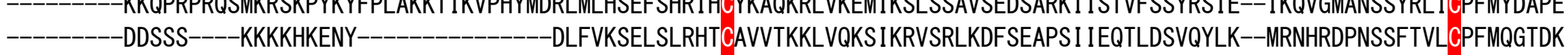

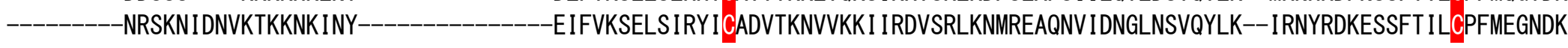
$:: *: \quad * \quad:: *::$

AEKMRAARAEMVTYAM------AKASTGK-----RLKRMLQKVRSWIRKKAFA--AARKLKSLKNRISTAFGRGKPP--KAKVPDWAVVNAGVG----MWTGKVFSTDLTFNEDEMSCDG ELR-NIHQLNI IKYVISKAVSKARFSPERKRLLTSLQSQPHKLYPT I QMEPLADLPGGVLVGTRKYNGVSYSGGYAPLEKI IYYPDNVVVKPGEG--RLVYDGSGFVPELMALRETVNVES HIR-DLERSQI ISYVL-KNVGIYNLFKGK---LHNLFSKSINIKEGVKSDSVV----TVKVGGRKFNGNLFVGGYNL--NVNDIDQNTLHIGLSKSRRVYNGTKFVDELDILKEE-GIKD NIR-ELERTQISLFIH-KNIGMSRI IKGK---LINIFKKTLNMREG IKSDSA I----SIKVGARKYNGI IFTGGYQL--NVDNLDQNTLHIGLSKTRKVYDGRKYVDELEILKGD-GVKN

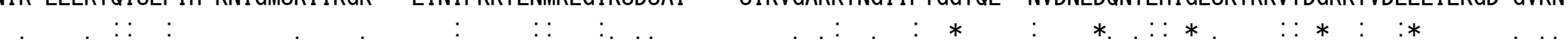

PHEPIRVMSWKQNHFTTFASSSTNAERNYVLVKKGD-----DSHCWATREALVHKG-------WSGIPVYQYAEPAGFWLQE-VSPSNQPFVVNWDG-YLTTSDNLTLQDI-----DINA IS--VRFEGGYNKYYYNMTDGTQVDERNFAIDSPD---FIVKSHVLTTANAL IKLGFDMGRI IWCG---YKHGWVADFVLSEVVGDTDVPV---FNGRYWLLSRELRVEDLVGPDMKIKI I--VKGIDEDNERLYVLSTGERVSEFDYAKENPNANI I IFDGNNYISSYALREI GLENERIVWAG---NTVGWTAEFALGN-I SDRPIPI---FDGSAWILLDKLSIKNILGNFLPKNV IY--MKGLNEDNERI YELQNNMRVSEFDYAI QNPDANI IVFDGNNY I SSYALRNMGLEHERIVWAG---PSVGWTAEFALSA-I SDNPLPI---FDGSAWVLLEKLSIRSILGKHLPSDV

SSDSLK----SHAVMRIVDSNGKTIY----------------QGPPTGVVQTQGGVVTLGSIRNLVSGVHSTGDSVEVRVTASGPQLTSVADLDTQFKEIPDL DGDEIKMENIDKYNIRIVNTNNEVMNLNKDSNKESSQSGDSPSNSAEGSFST ILGNI TPGGVKNLVKDASYIPERNTLVIDLDAP--_-_---DFVYSI GGNL NGNLLA----KDINFI ILNKDGKAIL----_---_---KN-TMPIVNLKNATFTLNG ILNFVIKAEK-GNDNEI I VHTRIPNGNSLA----NTVNFVILNKDGKPIL-KN-TTPVINLKYATFTLSGIVNFVIKAEK-GIGNEI IVHTRIP-

. : : : $: *::::$ :

$* \ldots: *: *$ 
Fig. S2. Western blot of mouse anti-PyRON2 serum and rabbit anti-PyAMA1 antibody used in this study.

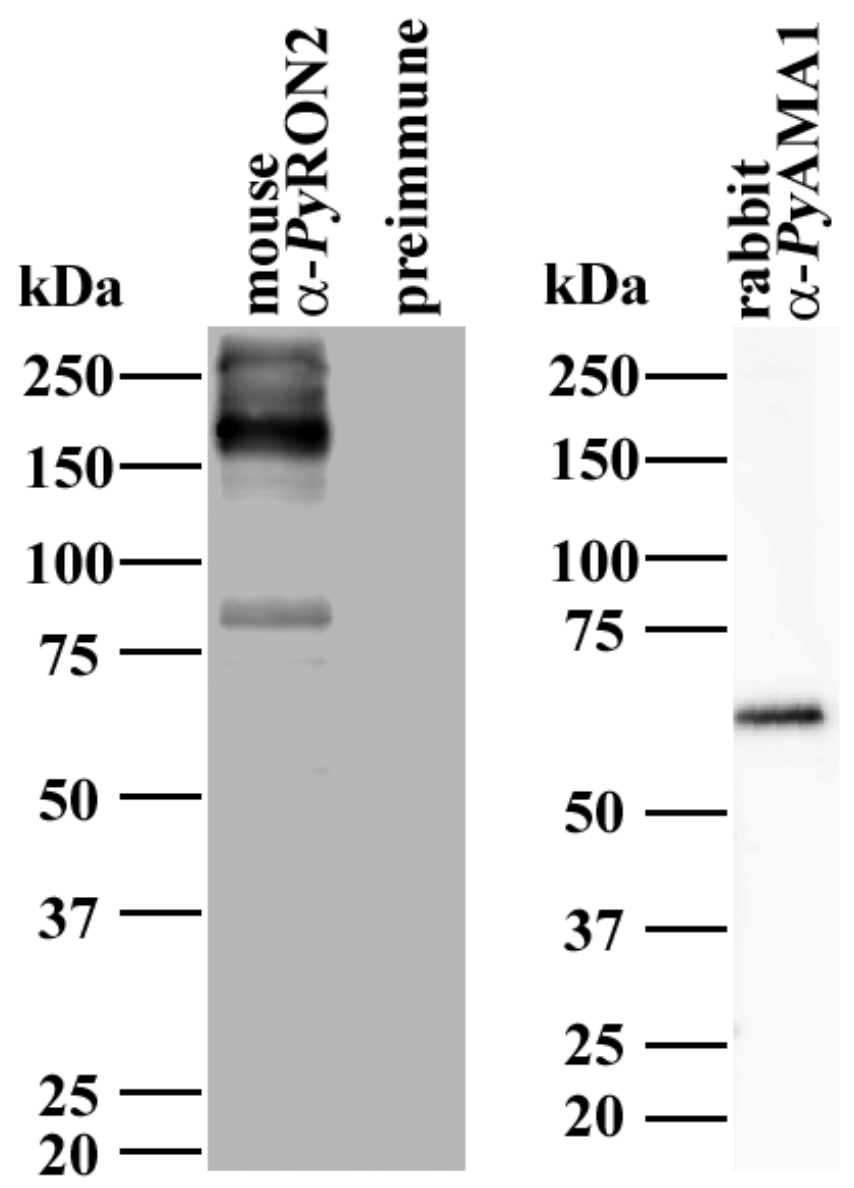


Figure S3. Indirect immunofluorescence assay with rabbit anti-PyRON5 crude serum and its preimmune serum and immunoelectron microscopy images with rabbit preimmune serum for anti-PyRON5.

(A)

\section{a-PyRON5}

crude serum

preimmune

serum

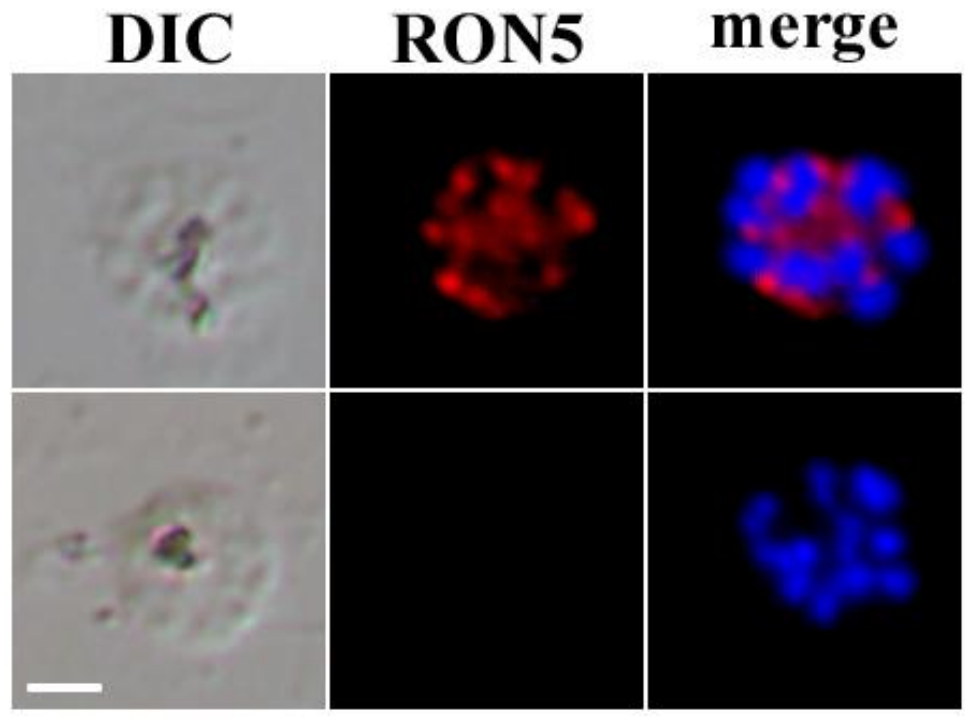

(B)

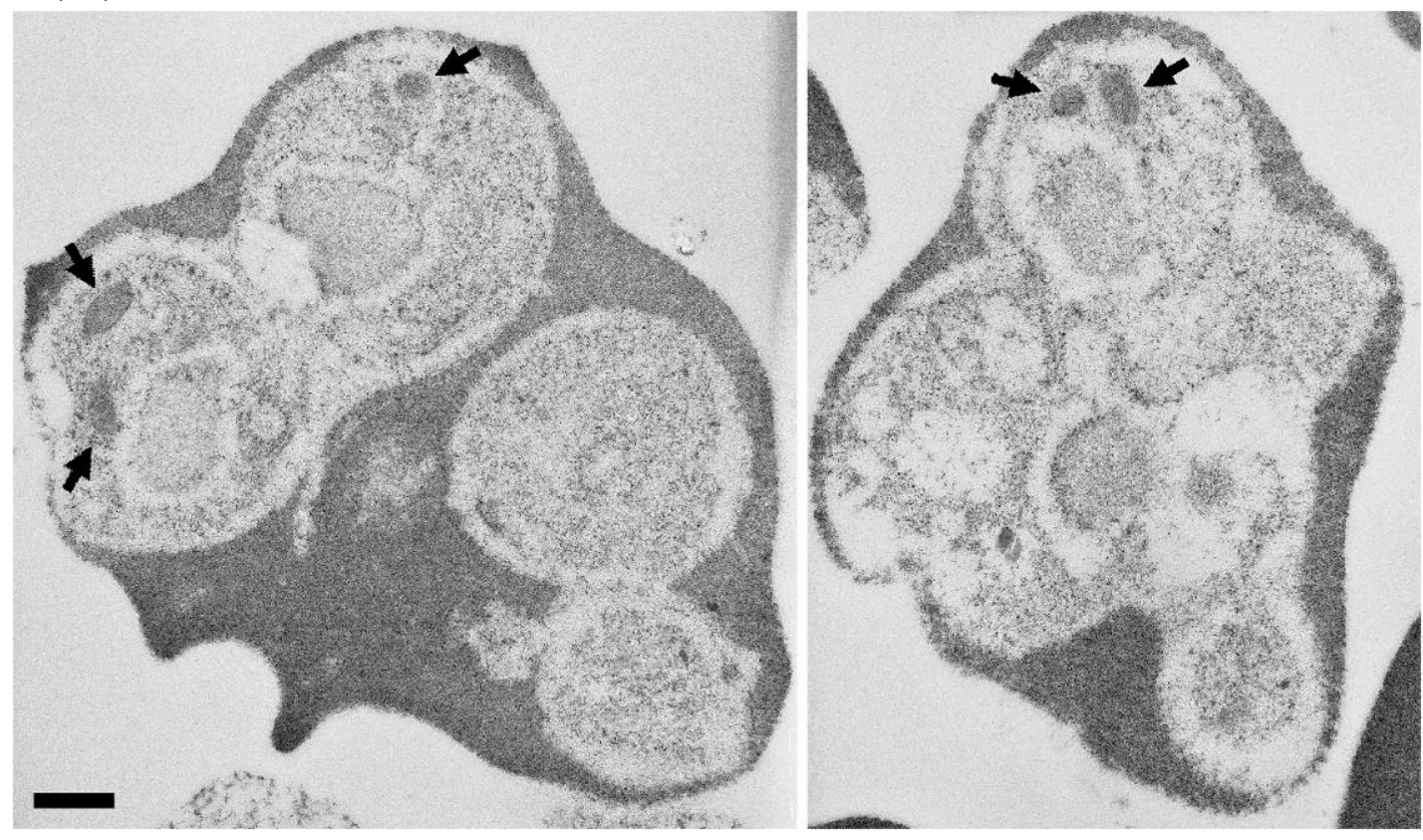

(C)

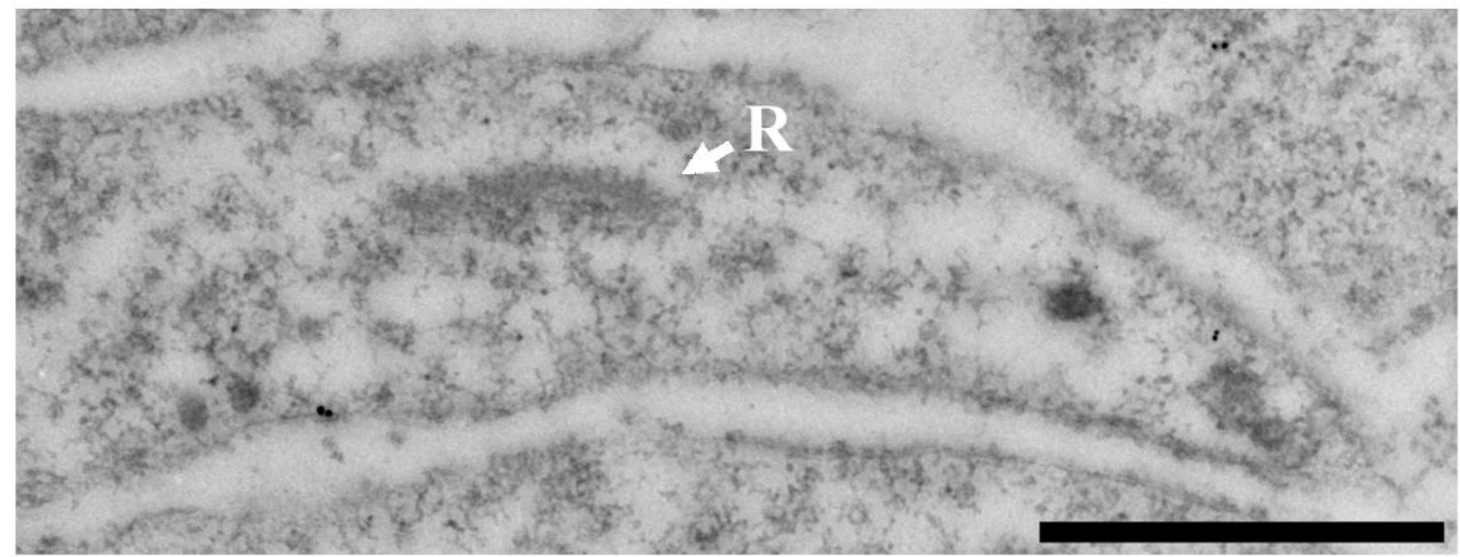


Figure S4. Primary protein structure of RON5 in Plasmodium yoelii, Plasmodium falciparum, and Toxoplasma gondii and schematics of the expected processing of PyRON5.

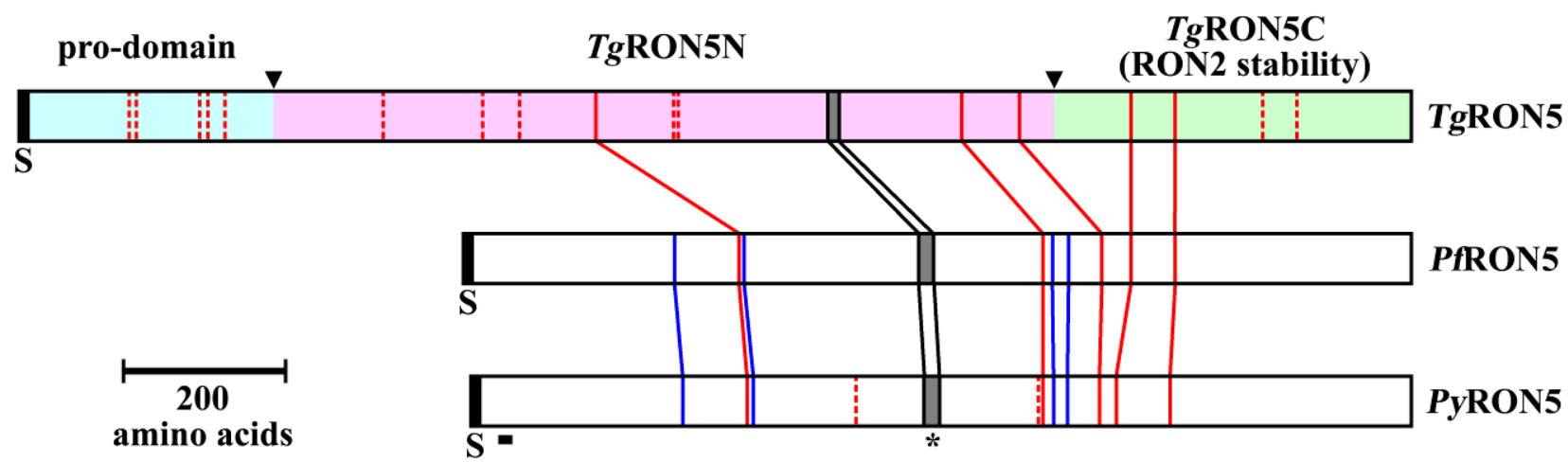

Expected processing of PyRON5

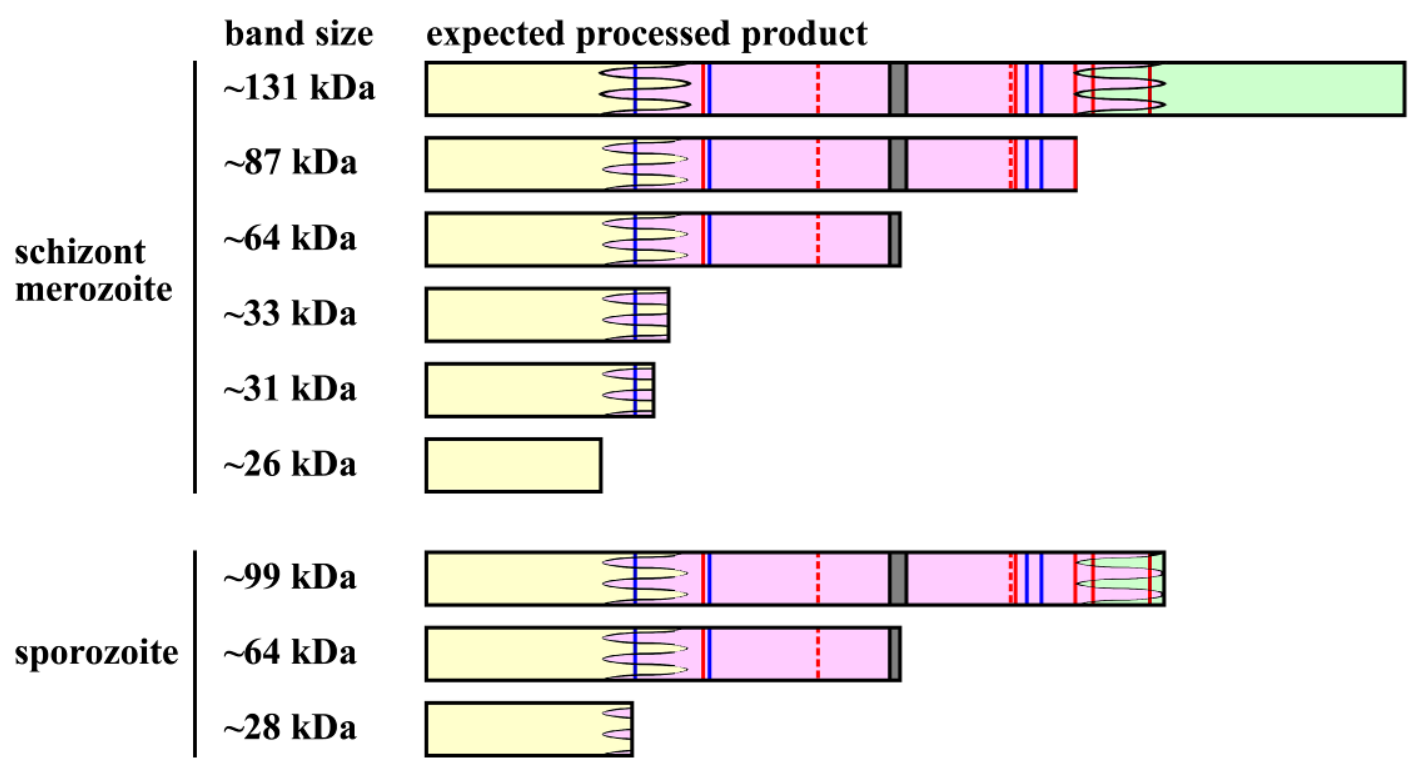

